\title{
La concordance bilingue grecque-syriaque des Discours de Grégoire de Nazianze
}

Par

\section{Bastien Kindt, Jean-Claude Haelewyck, Andrea Schmidt et Nicolas Atas}

Université catholique de Louvain

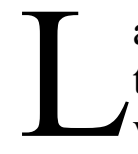

a concordance bilingue grecque-syriaque des œuvres de Grégoire de Nazianze présentée dans ces lignes ${ }^{1}$ fait partie d'un ensemble de développements expérimentaux relevant d'un projet plus ambitieux dont l'objectif est double.

1. Il s'agit, d'abord, de rendre accessibles en ligne, via une interface d'interrogation, les données lemmatisées publiées en 1990 et 1991 dans les deux volumes du Thesaurus Sancti Gregorii Nazianzeni, la première concordance grecque des opera omnia de Grégoire ${ }^{2}$. En utilisant cette interface, le chercheur peut obtenir, par exemple, toutes les occurrences du mot grec $\pi$ oun viv/pasteur attestées dans le corpus interrogé. Le résultat de cette requête s'affiche à l'écran sous la forme d'une concordance, comme illustré sous la figure $1^{3}$.

\footnotetext{
${ }^{1}$ Le contenu de cet article a fait l'objet d'une communication présentée par B. Kindt le 20 février 2017 dans le cadre du colloque Tradurre, tradire, tramandare i Padre Greci nell'Occidente Latino e nell'Oriente Siriaco 20-21 janvier 2017, Università degli Studi, Roma Tre.

2 Mossay et alii 1990 ; Mossay et alii 1991. Sur le Thesaurus Patrum Graecorum, une sous-série du Corpus Christianorum, cf. COULIE 1996, p. 35-54, et KINDT 2004, p. 213-237.

${ }^{3}$ Les figures de cet article sont également disponibles sur le site Internet du projet GREgORI, cf. https://uclouvain.be/fr/instituts-recherche/incal/ciol/gregori-project.html (les liens cités dans cet article étaient
} 
ToIfriv $\{\mathrm{N}+\mathrm{Com}\} 9$ GRNA Or. 1736400 D 1 GRNA Or. 1636400 B 1 GRNA OR. 1736401 A 10 GPNA Or. 13135853 A4 GPNA Or. $13335856 \mathrm{AB}$ GPNA Or. 13235 BS3 C 6 GRNA Or. 1735401 A 9 GRNA Or. 13435856 A 13 GRNA Or. 1635400 B 3

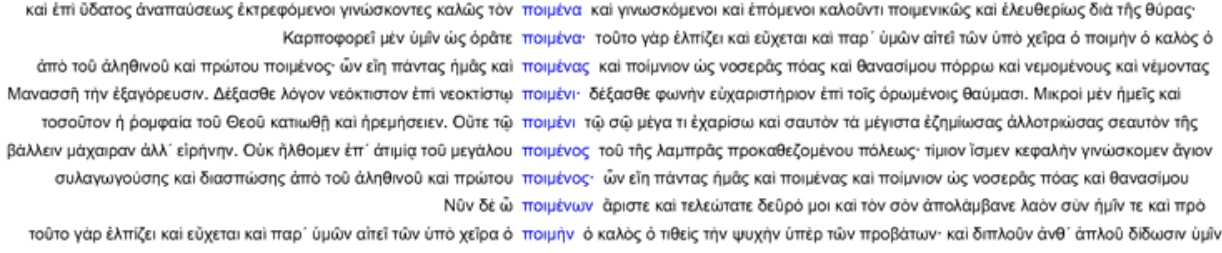

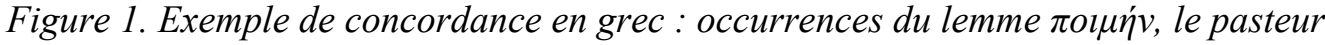

L'utilisateur peut réaliser des recherches basées sur un lemme, comme c'est le cas ici avec $\pi 0 u \mu \eta ́ v$. Ce type d'interrogation permet d'obtenir la concordance de toutes les formes fléchies

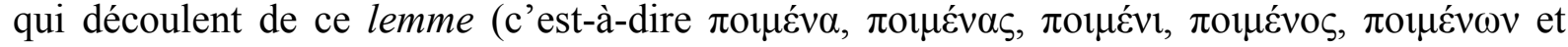

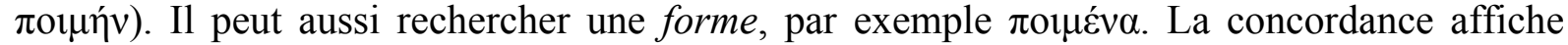

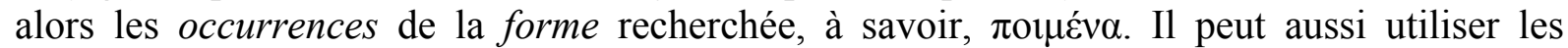
étiquettes identifiant la catégorie morphosyntaxique d'un lemme, par exemple, toujours pour $\pi \mathrm{o} \mu \eta \dot{v}$, « $\mathrm{N}+\mathrm{Com} »$, l'étiquette des noms communs. La concordance affiche dans ce cas toutes les occurrences des lemmes catégorisés comme noms communs. Enfin, il est également possible de baser les recherches sur une combinaison de ces différents éléments, afin de produire la concordance de toutes les séquences constituées, par exemple, d'un adjectif

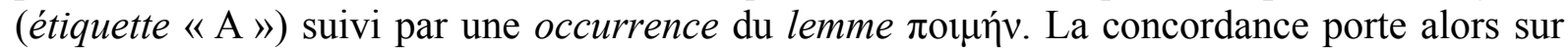

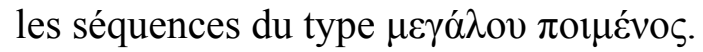

2. Il s'agit, ensuite, de mettre en parallèle les données lemmatisées du texte grec (le texte source) et celles des versions orientales - en arménien, en géorgien, en arabe et en syriaque ${ }^{4}-$, ces versions orientales étant les traductions anciennes des œuvres de Grégoire (les textes cibles). Pour arriver à ce résultat, deux opérations sont nécessaires; la lemmatisation des

actifs en date du 31 mai 2018). Le lecteur y trouvera aussi les versions complètes des concordances monolingues, en grec ou en syriaque, des Discours 1 et 13, ainsi que les concordances bilingues.

${ }^{4}$ Pour les développements en arménien, cf., par exemple, VAN ELVERDINGHE 2018. En géorgien, cf. CoULIE et alii 2013, p. 161-201, et PATARIDZE, KInDT 2018. Pour l'arabe, cf. TuERLinckX 2004, p. 1069-1078. En syriaque, cf. KINDT 2018. 
textes des versions orientales, d'une part, et l'alignement des textes sources et des textes cibles, d'autre part.

La lemmatisation des textes des versions orientales est en cours. Ainsi, pour le syriaque, l'interface permet de rechercher, comme dans le cas du grec illustré ci-dessus, les attestations du lemme $i$ ipasteur. L'utilisateur peut également formuler des interrogations portant sur une forme ou sur une étiquette relative aux catégories morphosyntaxiques, mais aussi sur la racine sémitique des mots ou sur une séquence de ces différents éléments. Le résultat s'affiche sous la forme d'une concordance, comme illustré sous la figure 2.

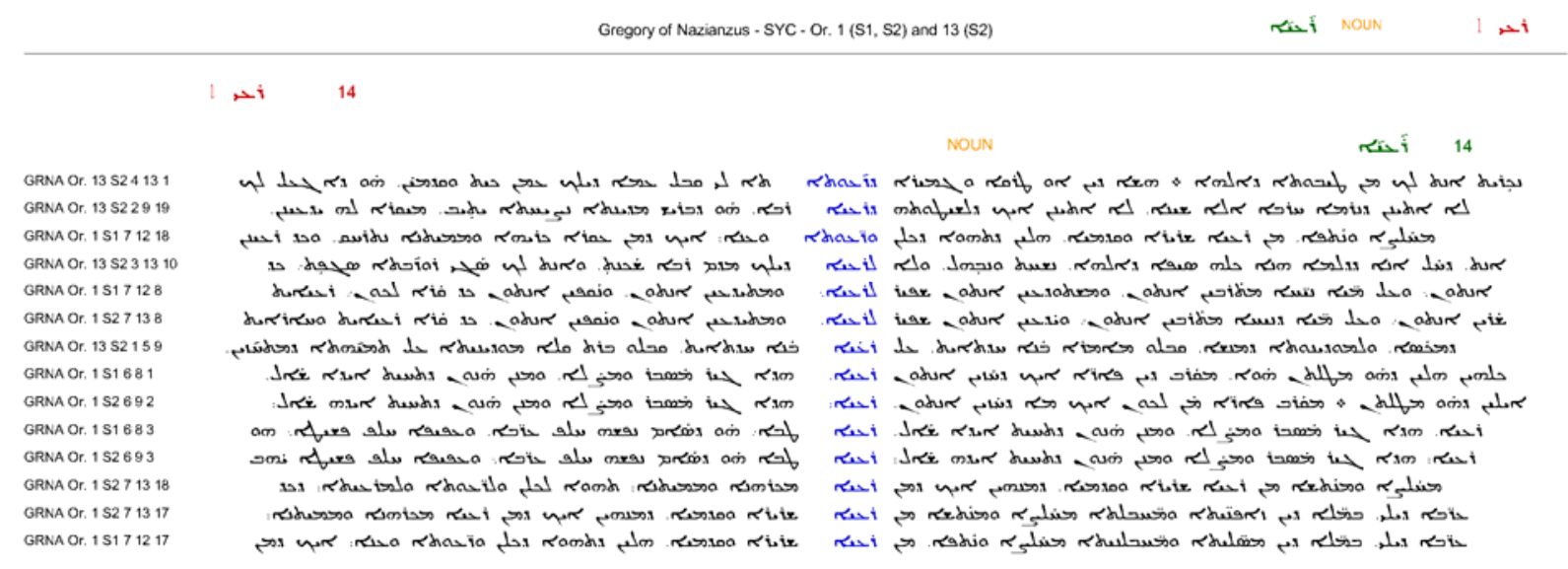

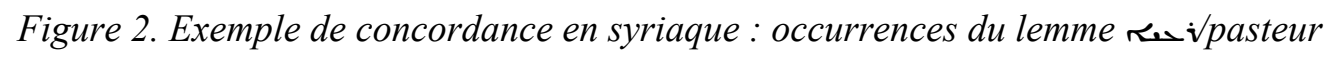

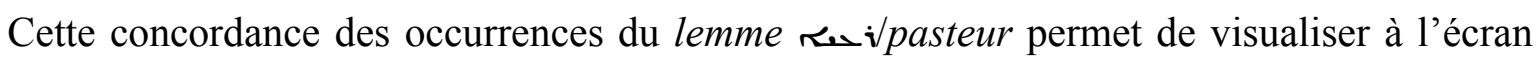
non seulement les formes simples du mot, mais aussi les formes suffixées du type ה. ה rail.

L'opération d'alignement, quant à elle, permet d'apparier les mots ou les expressions du texte source aux mots ou expressions qui en sont les traductions dans les textes cibles $^{5}$. Ainsi, l'utilisateur de l'interface peut interroger en même temps le texte grec et une de ses versions. Les résultats s'affichent alors sous la forme de concordances bilingues. Dans la concordance bilingue en grec et en syriaque, la requête basée sur le lemme grec $\pi$ ouńv fournit aussi les occurrences syriaques du lemme rivi.

\footnotetext{
${ }^{5}$ Sur les notions d'alignement, de texte source et de texte cible, cf. LEFER à paraître. À ce stade des développements, l'alignement est réalisé manuellement à l'aide du logiciel mkAlign développé à l'Université de Paris 3 par Serge Fleury : cf. FLEURY 2012.
} 
Gregory of Nazianzus - Ot. 1 - GRC-S1

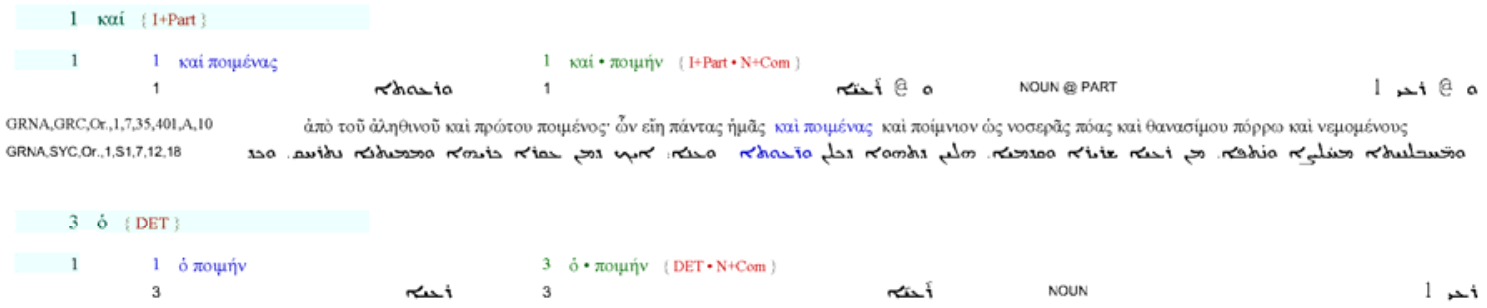

GRNA,GRC,Or.,1,635,400,B,3

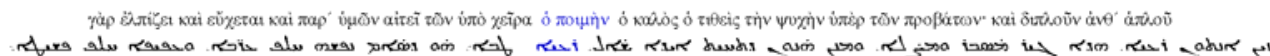

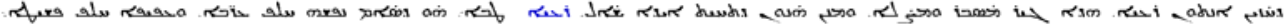

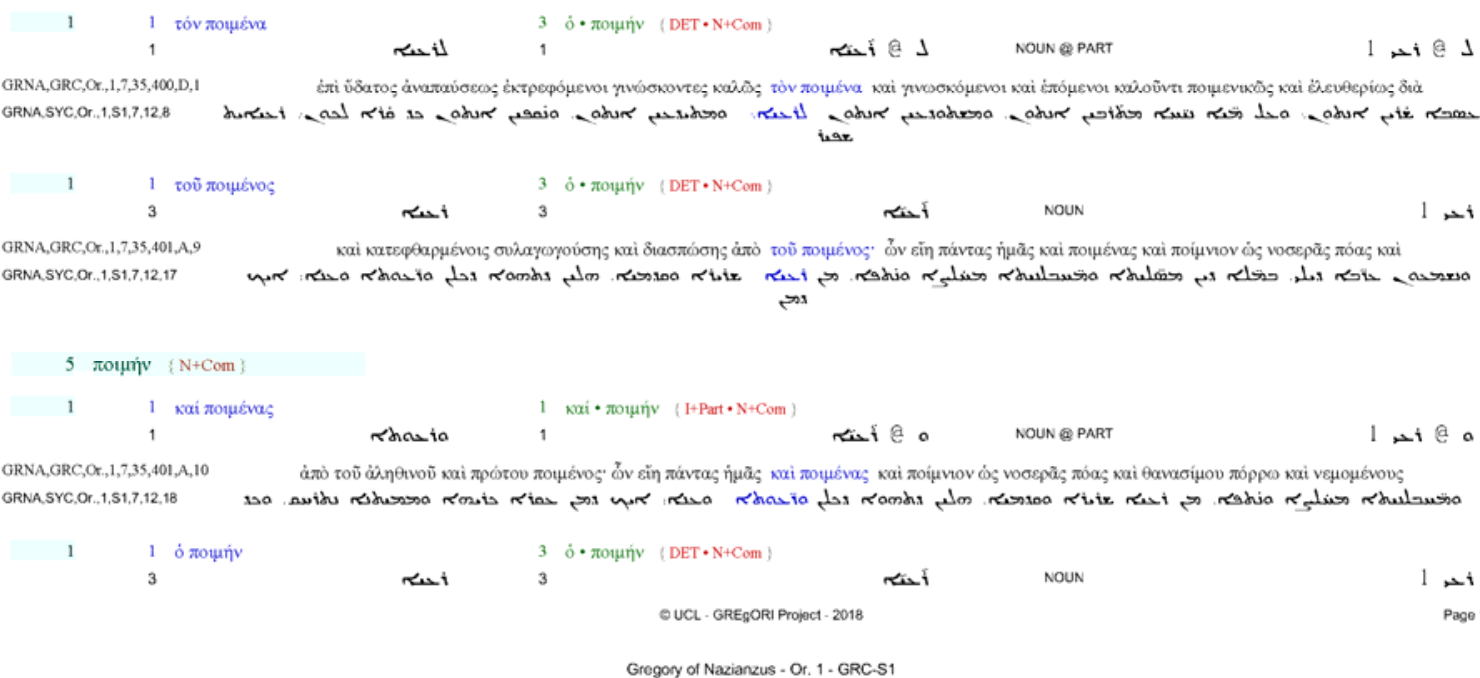

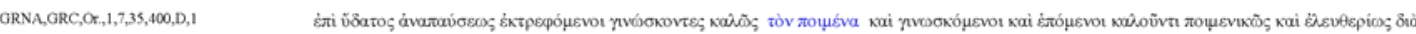

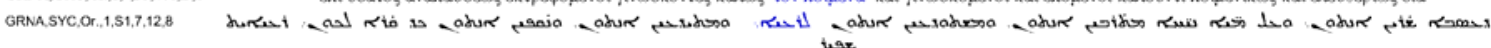

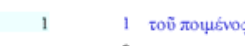

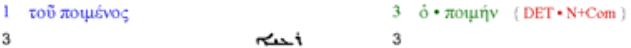
NA,GRC, $0 x, 1,7,35,401, A, 9$

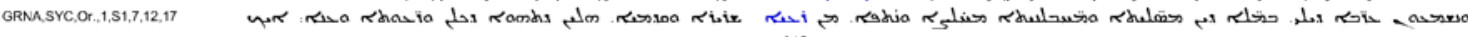

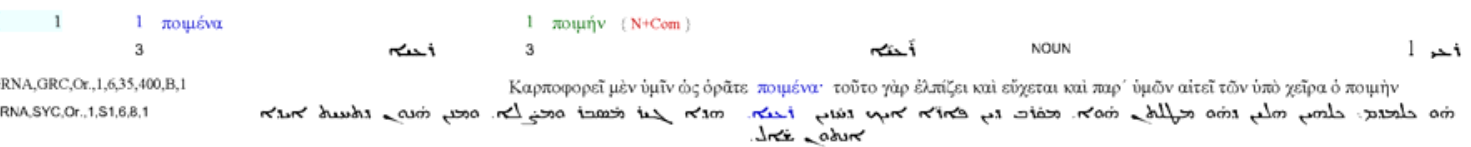

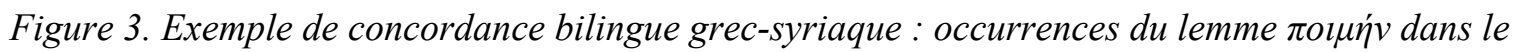
texte source et ses équivalents dans le texte cible 
L'ambition finale est d'offrir aux chercheurs un outil permettant d'interroger les textes grecs et leurs versions orientales dans un environnement simple d'utilisation, malgré la présence concomitante de sources écrites dans les différentes langues de l'Orient chrétien. Cela impose de mettre au point un outil intégrant de manière efficace l'affichage, sur un même écran de recherche, des différents alphabets utilisés pour écrire ces langues - en l'occurrence, ici, les alphabets latin, grec et syriaque - et des différents sens d'écriture - en l'occurrence horizontal dextroverse (de gauche à droite) pour le grec ou horizontal sinistroverse (de droite à gauche) pour le syriaque.

Ces travaux sont le fruit d'une collaboration entre le projet GREgORI, mené à l'Institut orientaliste de l'Université catholique de Louvain, et le CENTAL, Centre de traitement automatique du langage, un laboratoire d'informatique-linguistique de la même université ${ }^{6}$. Les philologues de l'Institut orientaliste conçoivent les ressources linguistiques utiles pour le traitement des langues abordées. Les informaticiens du CENTAL développent les outils informatiques permettant d'assurer la gestion, la visualisation et l'exploitation de ces données.

Nous exposons ici les résultats du traitement, en grec et en syriaque, des Discours 1 et 13 du Nazianzène. Après avoir décrit le corpus traité (Section 1), nous présentons les règles d'analyse établies pour la lemmatisation des textes syriaques, à l'instar de ce qui existe pour le $\operatorname{grec}^{7}$ (Section 2). Nous décrivons ensuite les concordances et différents outils lexicographiques produits avec les données lemmatisées (Section 3). Nous terminons par une description de la version expérimentale de l'interface en ligne (Section 4). Nous pourrons enfin conclure et ouvrir quelques perspectives (Section 5).

\section{Le corpus}

Le corpus analysé est constitué de deux homélies de Grégoire de Nazianze, les Discours 1

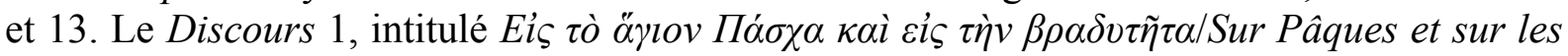
lenteurs de l'auteur, a été prononcé le jour de Pâques 362, le $31 \operatorname{mars}^{8}$; Le Discours 13 a

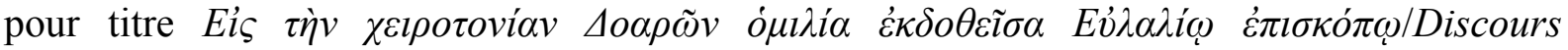
prononcé à Doara à l'occasion de la consécration de l'évêque Eulalios et se situe chronologiquement aux alentours de l'année $373^{9}$.

Pour le grec, les données lemmatisées sont directement reprises du Thesaurus cité. Le tableau 1 présente le nombre d'occurrences, de formes et de lemmes dans le corpus grec.

\begin{tabular}{|l|r|r|r|r|}
\hline & Occurrences & Formes différentes & Lemmes & Racines \\
\hline Discours 1 & 777 & 454 & 340 & - \\
\hline Discours 13 & 599 & 383 & 302 & - \\
\hline Total & 1376 & 764 & 559 & - \\
\hline
\end{tabular}

Tableau 1. Nombre d'occurrences, de formes différentes et de lemmes dans le corpus grec

\footnotetext{
${ }^{6}$ La description et la bibliographie complète du projet GREgORI sont accessibles sur le site Internet cité note 3. Pour le CENTAL, cf. https://uclouvain.be/fr/instituts-recherche/ilc/cental. Les auteurs tiennent à adresser leurs plus vifs remerciements à MM. Hubert Naets et Claude Devis, informaticiens au CENTAL.

${ }^{7}$ KINDT 2004, p. 237-271.

${ }^{8}$ Patrologia Graeca, 35, col. 395a-401b; lemmatisation : Bernard Coulie, Bastien Kindt et Justin Mossay†.

${ }^{9}$ Patrologia Graeca, 35, col. 852a-856c; lemmatisation : Bernard Coulie, Bastien Kindt et Justin Mossay $\dagger$.
} 
Pour le syriaque, l'analyse porte sur les textes publiés dans le Corpus Nazianzenum ${ }^{10}$ et a été réalisée ab ovo. La version syriaque des homélies de Grégoire est constituée de plusieurs états de traduction ${ }^{11}$. Le premier, une Versio antiqua nommée $\mathrm{S} 1$, date du $\mathrm{VI}^{\mathrm{e}}$ siècle. Le second relève d'une traduction dite « intermédiaire », une Versio media nommée Sm et située chronologiquement après 550. Le troisième, une Versio nova nommée S2, est une révision de $\mathrm{S} 1$ et de Sm réalisée par Paul d'Édesse au VII ${ }^{\mathrm{e}}$ siècle. Les éditions des Discours 1 et 13 reprennent $\mathrm{S} 1, \mathrm{Sm}$ et $\mathrm{S} 2^{12}$. Le tableau 2 présente le nombre d'occurrences, de formes, de lemmes et de racines dans le corpus syriaque.

\begin{tabular}{|c|c|c|c|c|}
\hline & Occurrences & $\begin{array}{c}\text { Formes } \\
\text { différentes }\end{array}$ & Lemmes & Racines \\
\hline Discours 1 (S1) & 685 & 475 & 323 & 262 \\
\hline Discours 1 (S2) & 711 & 483 & 326 & 263 \\
\hline Discours 13 (S2) & 560 & 398 & 289 & 254 \\
\hline Total & 1956 & 947 & 562 & 421 \\
\hline
\end{tabular}

Tableau 2. Nombre d'occurrences, de formes différentes, de lemmes et de racines dans le corpus syriaque

Ce corpus bilingue réunit un ensemble de données quantitativement modestes. De fait, en grec, les homélies de Grégoire totalisent plus de 216.000 occurrences et, en syriaque, le corpus des textes déjà publiés dans le Corpus Nazianzenum atteint les 103.000 occurrences. L'analyse proposée ici pour le syriaque ne prétend donc pas fournir une description globale et aboutie de la langue syriaque, ni de manière générale, ni en ce qui concerne plus particulièrement la langue des différents états des versions syriaques de Grégoire. Le but est, plus modestement, d'offrir une assise expérimentale à une analyse cohérente et stable des textes, dans le cadre d'un projet plus large de lemmatisation et d'alignement des textes sources et des textes cibles. Il s'agit donc de définir, pour la lemmatisation en syriaque, des principes directeurs, comme cela a été fait, in illo tempore, pour le $\operatorname{grec}^{13}$. Les questions principales portent sur l'intitulé des lemmes et des racines, et sur la définition des catégories morphosyntaxiques dont relèvent les lemmes. Ces principes, simples, cohérents et stables - censés décrire sans trahir - pourront servir de normes pour les analyses futures menées tant par les membres du projet GREgORI que par des collaborateurs extérieurs.

\section{Ressources linguistiques pour la lemmatisation du syriaque}

En grec, la lemmatisation permet d'identifier le lemme de chacune des formes présentes dans un texte donné. De plus, chaque lemme est accompagné d'une indication de sa catégorie morphosyntaxique (nom, verbe, adjectif, pronom, etc.), la partie du discours dont le lemme relève. L'utilisateur travaillant sur une interface en ligne peut ainsi rechercher dans les textes

${ }^{10}$ Pour le Discours 1: HaElewYCK 2011 (Versio Antiqua [S1], p. 2-12; Versio nova [S2], p. 3-13); lemmatisation : Jean-Claude Haelewyck, Bastien Kindt, Andrea Schmidt, avec la collaboration de Nicolas Atas ; alignement: Jean-Claude Haelewyck, Bastien Kindt. Pour le Discours 13 : ScHMIDT 2002 (Versio nova [S2], p. 3-15); lemmatisation: Naima Afif, Jean-Claude Haelewyck, Bastien Kindt, avec la collaboration de Nicolas Atas ; alignement : Jean-Claude Haelewyck, Bastien Kindt.

${ }^{11}$ Sur les versions syriaque des Discours de Grégoire de Nazianze, cf. HAELEWYCK 2017b, p. 75-124.

${ }^{12}$ Les différents Discours ne sont pas tous attestés dans ces trois états. Ainsi, pour le Discours 13, la version Sm n'est pas attestée.

${ }^{13}$ Voir la bibliographie citée aux notes 2 et 4. 
une forme, mais aussi un lemme ou encore tous les lemmes relevant d'une catégorie morphosyntaxique donnée, ou, enfin, une combinaison de ces différents éléments. Les réponses correspondant à ces recherches s'affichent à l'écran, instantanément, sous la forme d'une concordance énumérant la totalité des occurrences répondant à la requête formulée par l'utilisateur. Dans ces concordances, chaque forme est accompagnée de sa référence dans l'édition utilisée et encadrée d'un large contexte.

La même approche est utilisée pour l'analyse du syriaque, moyennant d'indispensables adaptations dues aux particularités de cette langue par rapport au grec. Deux particularités peuvent être citées d'emblée: l'abondance de formes suffixées et préfixées, et la présence d'une unité lexicale placée hiérarchiquement au-dessus du lemme, à savoir la racine sémitique des mots. Ces éléments du lexique doivent être définis.

\subsection{Les formes}

Les textes traités correspondent aux textes édités, considérés comme textes de base. Dans la mesure du possible, un texte de base est la version numérique d'un texte ayant fait l'objet, comme c'est ici le cas, d'une édition critique. Les occurrences sans vocalisation ou avec une

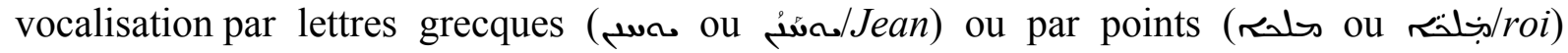
conservent la graphie qui est la leur dans le texte de base.

Les occurrences constituées de plusieurs formes - réunissant dans une seule unité graphique plusieurs unités lexicales, y compris les éléments préfixés et suffixés - sont traitées de manière à rendre possible l'identification des différentes formes concernées. Ainsi, l'occur-

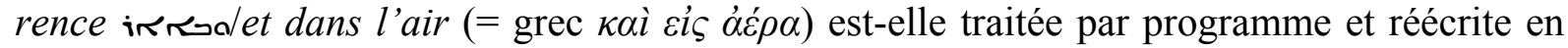
troisformes distinctes irk@,@a, chacune séparée par le signe «@», ce qui rend explicite, dans la suite des traitements, la continuité des lexèmes en présence, à savoir: (1) o, la particule conjonctive ; (2) $\neg$, la particule prépositionnelle; (3) i $\kappa \kappa$, le nom. La représentation des lemmes, des catégories morphosyntaxiques et des racines en présence dans cette occurrence repose sur le même principe: (1) lemmes irk@,@o; (2) catégories NOUN@PART@PART; (3) racines irr@_@a.

Ce type d'analyse est directement inspiré du traitement des crases du grec. Dans une crase, deux lexèmes sont réunis dans une seule occurrence, une seule unité graphique. L'occurrence

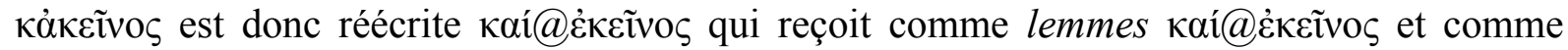
analyses morphosyntaxiques «I+Part@PRO+Dem», une particule (I+Part) suivie d'un démonstratif $(\mathrm{PRO}+\mathrm{Dem})^{14}$. La différence entre le grec et le syriaque est cependant sensible ; elle tient en deux points essentiels. D'abord, les formes préfixées et suffixés du syriaque sont quantitativement beaucoup plus représentées que les crases du $\operatorname{grec}^{15}$. Ensuite, les crases du grec sont toujours constituées de deux formes, alors qu'en syriaque, le nombre de formes réunies en une seule unité graphique peut être plus grand. Dans le corpus traité, plusieurs occurrences réunissent jusqu'à quatre éléments différents, comme par exemple, diosblavaba/et pourma pusillanimité (grec $\tau \tilde{\eta} \varsigma \varepsilon \dot{\varepsilon} \mu \tilde{\eta} \varsigma \delta \varepsilon i \lambda i \alpha \varsigma$ ), segmenté en,@hasbwa@」@a, séquence identifiant les quatre formes en présence, elles-mêmes classées sous les quatre lemmes attendusrr@rhoublow:」@o, eux-mêmes répertoriés sous les racines rro@1wa,@o.

\footnotetext{
${ }^{14}$ Chaque langue traitée dans le cadre du projet GREgORI à un jeu d'étiquette qui lui est propre. Les étiquettes utilisées pour l'analyse du syriaque peuvent donc être différentes de celle du grec.

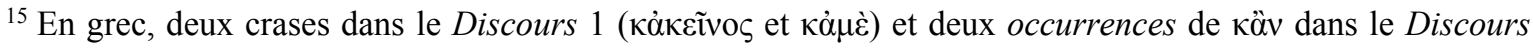
13. Dans le corpus syriaque, 778 occurrences (sur un total de 1956) sont préfixées ou suffixées.
} 
Il est important de noter que ces modifications ne s'appliquent qu'au texte traité et ne modifient en aucun cas la version originale du texte édité. Dans les concordances et autres outils lexicographiques, les occurrences sont reprises telles qu'elles apparaissent dans les textes originaux. Par contre, elles sont reprises sous chacun des lemmes dont elles relèvent. Dans une concordance, l'occurrence ir a figure donc à la fois sous le lemme ir lemme $\_$et sous le lemme a.

Certaines occurrences considérées comme figées sont analysées comme des formes simples. C'est le cas de r.moulseul, seulement (et non rsw@J@د) ou de pida/ensuite (et non (@id ). Ces cas, peu nombreux, seront systématiquement inventoriés dans un document à l'usage des collaborateurs.

\subsection{Les lemmes}

Le lemme peut se définir comme une "forme de départ» ou une "forme de regroupement » dont découlent les formes (fléchies) relevant d'un même paradigme ; le lemme représente ce paradigme. Il est aussi « une forme neutralisée du discours métalinguistique » servant à la description du lexique d'une langue ; en théorie, mais pas obligatoirement, le lemme correspond à une forme attestée $\mathrm{e}^{16}$.

Par convention, en syriaque, l'intitulé des lemmes est formulé à l'état emphatique puisque celui-ci s'est généralisé au détriment de l'état absolu: la forme 2 rost classée sous le

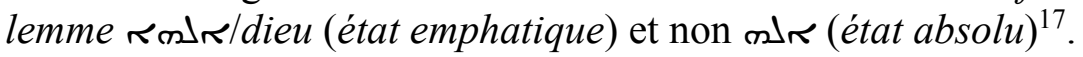

Même si en syriaque les noms et les adjectifs relèvent de paradigmes flexionnels identiques, ils sont rationnalisés sous deux lemmes différents : nom et adjectif. Ainsi la forme rho/maison (en grec oĩkos) est lemmatisée rhø, un nom, tandis que la forme

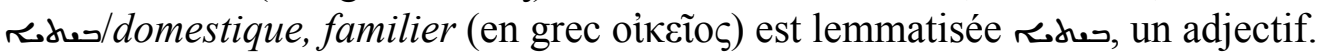

Les formes au pluriel ont, par convention, un lemme au singulier. Mais les plurale tantum

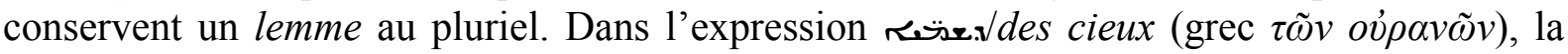

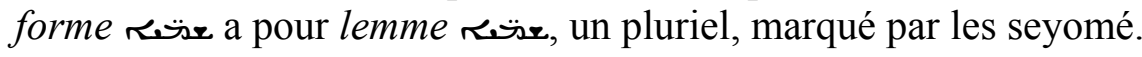

Les formes des composés véritablement lexicalisés en syriaque sont classées sous un lemme unique. Ainsi les formes du composé حשi/homme (littéralement fils d'homme) ne sont pas classées sous les deux lemmes כוֹ/fils et véritablement lexicalisé en syriaque puisqu'en dérivent le verbe dénominatif घiऽłr/devenir homme, s'incarner et le substantif rharsistos/incarnation ${ }^{18}$. Il en va de même pour les

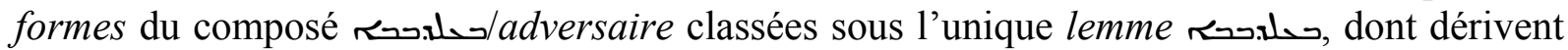

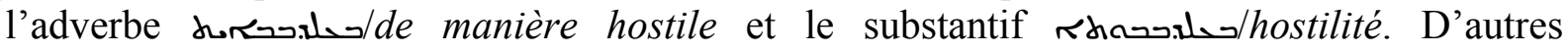
composés, qui n'ont pas été lexicalisés en syriaque, tels que حلsoltoute chose et

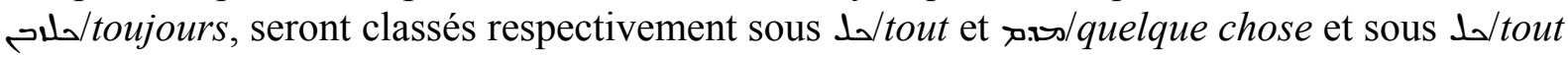
et rivitemps.

\footnotetext{
${ }^{16}$ Pour une définition du lemme, cf. KINDT 2004, p. 220-221, § 1-3.

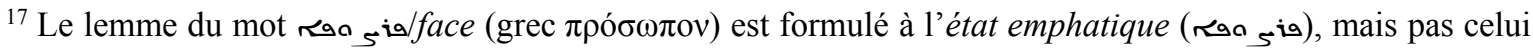
du mot ir /air (grec ợ̂), ce qui obligerait à créer un lemme artificiel «irর. En réalité, les mots grecs qui ne finissent pas en -o n'ont pas de variations morphologiques pour les états ; cette forme ir $r$ peut donc être à l'état absolu, construit ou emphatique.

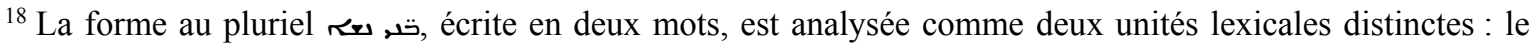
lemme riv et le lemme revr.
} 
Les formes féminines des adjectifs reçoivent, toujours par convention, un lemme masculin. Ainsi, la forme rhlew. est-elle classée sous le lemme r.w./redoutable, terrible; la forme rhلsir/veuve est classée sous le lemme مar/femelle qui, par nature, n'a pas de masculin, est laissé au féminin.

Les verbes à troisième radicale faible reçoivent une racine à troisième yod, à l'exception des six verbes troisième aleph qui maintiennent l'aleph comme gutturale ${ }^{19}$. Pour le lemme de ces verbes à $3^{\mathrm{e}}$ yod, on ne fait pas la distinction entre verbes d'action ( $3^{\mathrm{e}}$ aleph au pe $\mathrm{c}$ al parfait) et verbes d'état (en général $3^{\mathrm{e}}$ yod au pe'al parfait). La forme avec $3^{\mathrm{e}}$ 'aleph servira donc systématiquement de lemme, ainsi וحم et non וحص être pur 》.

En cas de graphie multiple, la graphie la plus habituelle sert de lemme. Les formes حل

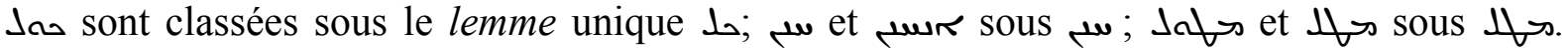
Quand la statistique ne permet pas de déterminer l'intitulé du lemme, on suit le dictionnaire de référence, en l'occurrence le Syriac Lexicon de M. Sokoloff; les formes ot rharche sont ainsi réunies sous le lemme soient pas enregistrés dans les dictionnaires usuels de la langue syriaque; dans ce cas, le nouveau lemme est créé et consigné dans une liste de mots dits « athesauristus ».

Les lemmes homographes - même si un ou plusieurs des lexèmes qu'ils représentent n'est ou ne sont pas attestés dans le texte traité- sont distingués par les points diacritiques, par la vocalisation par points ou par la linea occultans, comme dans les exemples suivants :

ařlou, ou bien vs ār/ô vs ar/oh!, hélas! ; , (pronom personnel indépendant et suffixé) vs

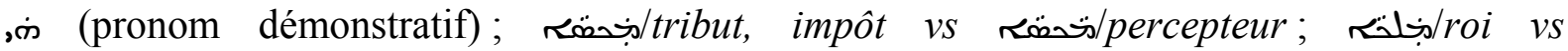

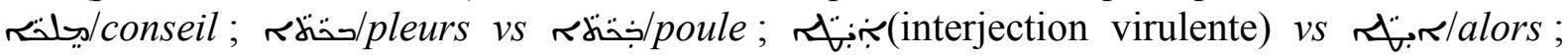

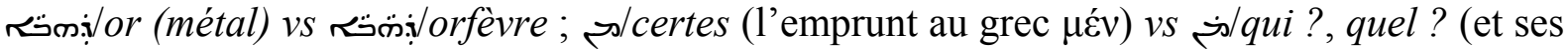
différents emplois) $v s$ plà partir de.

Pour assurer un traitement informatique homogène de ces mots, il est indispensable de vérifier que, dans les textes traités, un point-voyelle ne soit pas noté pour un point diacritique, ou inversement, et de respecter le standard d'encodage UNICODE ${ }^{20}$, tel qu'illustré dans le tableau 3.

\begin{tabular}{|c|c|c|c|}
\hline & \multicolumn{2}{|c|}{ Exemples } & Codage UNICODE \\
\hline Point-voyelle supérieur (MAJ+B) & $\dot{r}$ & i آلمّله & $\mathrm{U}+073 \mathrm{~F}$ \\
\hline Point-voyelle inférieur (MAJ+V) & $r$ & حبiiذيم & $\mathrm{U}+073 \mathrm{C}$ \\
\hline Point-diacritique supérieur (MAJ+P) & $\dot{r}$ & वக் & $\mathrm{U}+0307$ \\
\hline Point-diacritique inférieur (MAJ+M) & $\kappa$ & s & $\mathrm{U}+0327$ \\
\hline
\end{tabular}

Tableau 3. Codage Unicode des points-voyelles et des points-diacritiques

\subsection{Les catégories morphosyntaxiques}

Chaque lemme reçoit une étiquette indiquant la catégorie morphosyntaxique, la partie du discours, dont il relève. Cette étiquette caractérise le lemme au niveau du lexique en général.

${ }^{19}$ Il s'agit des verbes suivants: 2 /consoler,

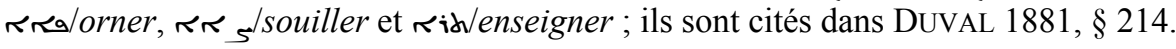

${ }^{20}$ Pour un complément d'information, cf. le site du Consortium Unicode accessible sous l'adresse http:/unicode.org/charts/ ainsi que le guide d'utilisation des polices de caractères Meltho rédigé par George Kiraz et accessible sur le site de la fondation Beth Mardutho sous l'adresse http://www.bethmardutho.org/index.php/resources/fonts.html. 
Elle ne décrit en aucun cas l'emploi particulier d'un lemme dans un contexte précis. Ainsi, dans l'expression suivante :

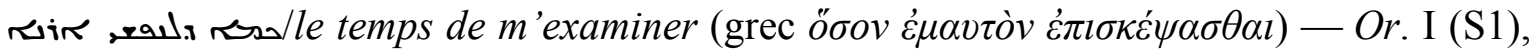
$\S 2,1.1-2$ (= HAELEWYCK 2011, p. 4),

la forme ra/âme - incluse dans l'occurrence, ravi - reste classée sous le lemme homographe r ras caractérisé comme nom, même si, dans ce contexte, elle rend, en français, l'équivalent d'un pronom réfléchi. De même, dans la phrase suivante :

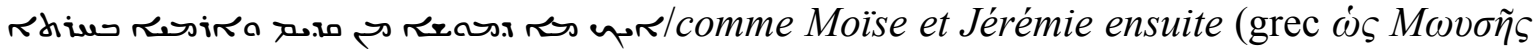

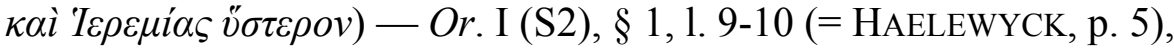

la séquence ${ }^{-}$: suite des lemmes $\backsim$, rogatif et particule.

Dans l'état actuel du projet, les étiquettes distinguent douze catégories morphosyntaxiques: ADJ, les adjectifs ; ADV, les adverbes ; CARD, les déterminants numériques cardinaux ; NAME, les noms propres ; NOUN, les substantifs ; ORD, les déterminants numériques ordinaux ; PART, les particules (regroupant les négations, les prépositions et d'autres mots invariables) ; PRO_dem, les pronoms démonstratifs ; PRO_ind, les pronoms indéfinis ; PRO_int, les pronoms interrogatifs ; PRO_pers, les pronoms personnels indépendants et suffixés; et enfin V1 à V28, les verbes. Ces catégories, classées selon l'ordre alphabétique des étiquettes, sont explicitées ci-dessous.

\subsubsection{ADJ = Les adjectifs}

(1)/les voici pour vous : Abraham le vénérable, ce patriarche, cette tête précieuse et respectée (grec $\tau \alpha \tilde{v} \tau \alpha \mu \dot{\varepsilon} v$ viñ $o$

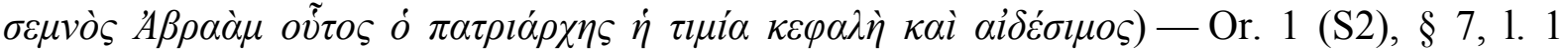
(= HAELEWYCK 2011, p. 11)

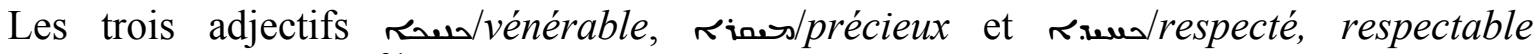
reçoivent l'étiquette $\mathrm{ADJ}^{21}$.

\subsubsection{ADV $=$ Les adverbes}

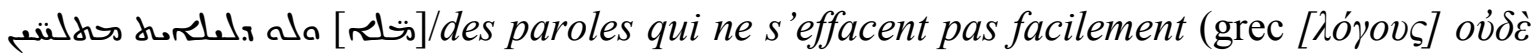

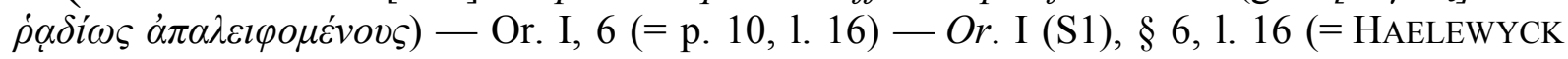
2011, p. 10)

Les adverbes reçoivent l'étiquette ADV; cf., par exemple, 丸へلlu/facilement;

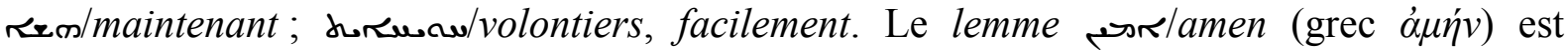
enregistré comme adverbe.

\footnotetext{
${ }^{21}$ De nombreux adjectifs sont à l'origine des participes. C'est le cas des trois adjectifs cités ici : حسبح et sont des participes pe'al passifs et l'étiquette ADJ. On suit en cela le Syriac Lexicon de M. Sokoloff (respectivement aux p. 617, 634 et 755) et le Dictionnaire syriaque-Français de L. Costaz (p. 144, 154 et 158). Le Thesaurus Syriacus (col. 1624, 1719) classe les deux premiers comme participes, et le troisième (col. 1765) comme adjectif. Le Compendious Syriac Dictionary de J. Payne Smith ne tranche pas pour le premier et le troisième en les désignant comme « part. adj. » (p. 196, 218), tandis que le second est vu comme un participe (p. 212).
} 


\subsubsection{CARD $=$ Les déterminants numériques cardinaux}

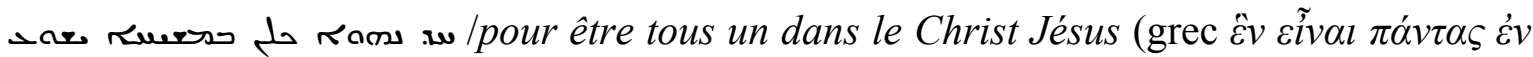

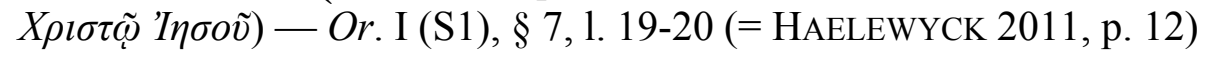

Les déterminants numériques cardinaux reçoivent l'étiquette CARD; cf., par exemple, .w/1; גل/ $/ 3 ;$ rod/100. Lemmes et racines revêtent une forme masculine ${ }^{22}$.

\subsubsection{NAME $=$ Les noms propres}

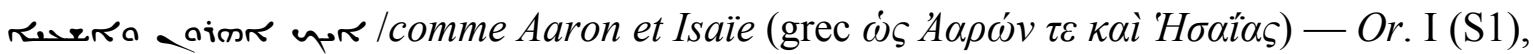
$\S 1,1.11$ (= HAELEWYCK 2011, p. 4)

(isc/aujourd'hui nous avons échappé totalement à l'Égypte (grec

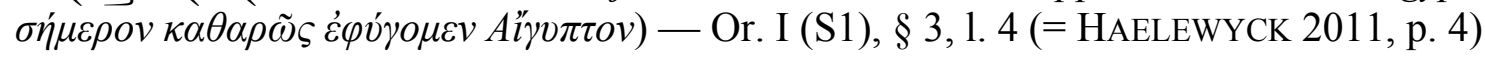

Les noms propres reçoivent l'étiquette NAME; cf., par exemple, ormis/Abraham;

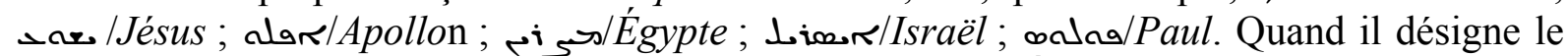
Christ, le lemme ruess est catégorisé comme nom propre. À ce stade du projet, l'étiquette NAME recouvre les anthroponymes et les toponymes.

\subsubsection{NOUN $=$ Les noms}

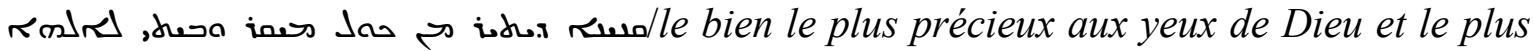

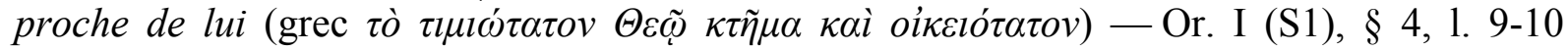
(= HAELEWYCK 2011, p. 6)

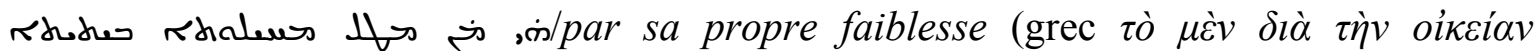

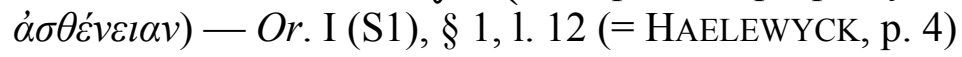

Les trois substantifs مسw/bien, $r$ /Dieu et rhalew/faiblesse reçoivent chacun l'étiquette NOUN.

\subsubsection{ORD $=$ Les déterminants numériques ordinaux}

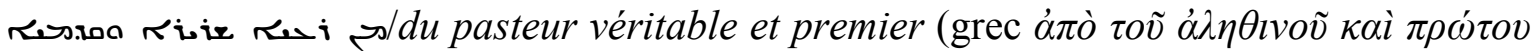
$\pi о \imath \operatorname{c} v o \varsigma)$ - Or. I (S1), § 7, 1.17 (= HAELEWYCK 2011, p. 12)

Les déterminants numériques ordinaux reçoivent l'étiquette ORD; cf., par exemple, ras.o/ $/ 1^{e r} ; \mathrm{ridh} / 2^{e}$.

\subsubsection{PART $=$ Les particules}

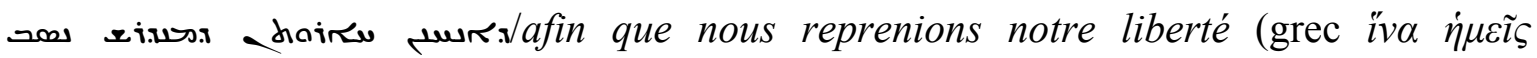
$\dot{\alpha} \pi \sigma \lambda \dot{\alpha} \beta \omega \mu \varepsilon v)-O r . \mathrm{I}(\mathrm{S} 1), \S 5,1.5$ (= HAELEWYCK 2011, p. 8)

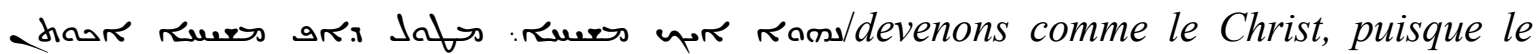

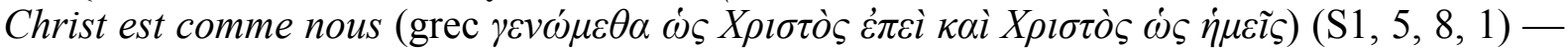
Or. I (S1), § 5, 1.1 (= HAELEWYCK 2011, p. 8)

\footnotetext{
${ }^{22}$ Même si, en raison de la polarité sémitique, certains d'entre eux (de 3 à 10) s'utilisent au masculin avec un substantif féminin et inversement.
} 
Les négations, les prépositions et certains mots invariables, éléments suffixés ou non, sont classés comme particules sous l'étiquette PART : ه/non, ne pas;

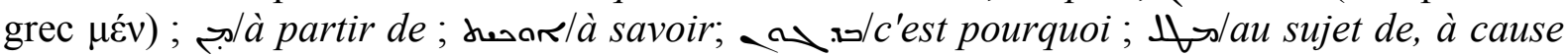
de; م. م.avant, devant; de même que la particule relative ou conjonctive. ou les interjections, ainsi $\kappa \mathrm{m} /$ voici. Ces éléments sont généralement considérés comme des conjonctions par $\mathrm{M}$. Sokoloff.

\subsubsection{PRO_dem = Les pronoms démonstratifs}

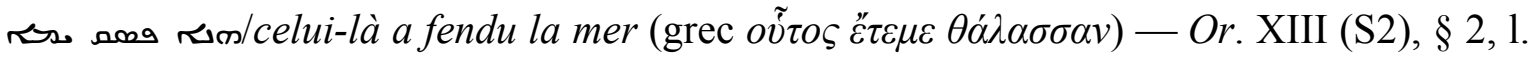
1 (= SCHMIDT 2002, p. 7)

Les pronoms démonstratifs reçoivent l'étiquette PRO_dem ; cf., par exemple, रım ; ari ; ,க் ; vm.

\subsubsection{PRO_ind $=$ Les pronoms indéfinis}

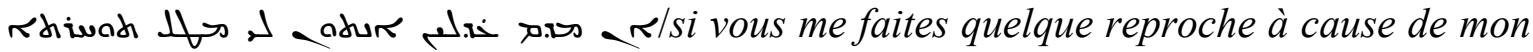

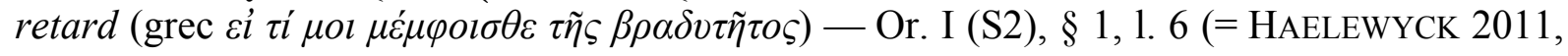
p. 3)

Les pronoms indéfinis sont classés sous l'étiquette PRO_ind, ainsi wive/autre, حلـ/tout, o. $/$ quelque chose, quelque ${ }^{23}$.

\subsubsection{PRO_int $=$ Les pronoms interrogatifs}

diris ar dur Or. XIII (S2), § 3, 1.1 (= SCHMIDT 2002, p. 11)

Les pronoms interrogatifs, cfr, par exemple, reçoivent l'étiquette $\mathrm{PRO}$ int, même quand ils sont utilisés, abusivement ${ }^{24}$, comme antécédents d'une proposition relative, comme dans la phrase suivante :

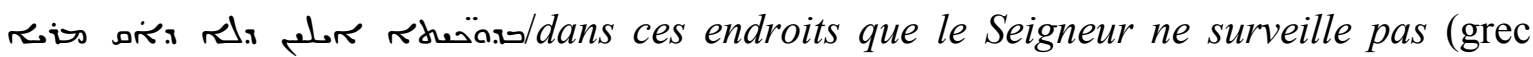

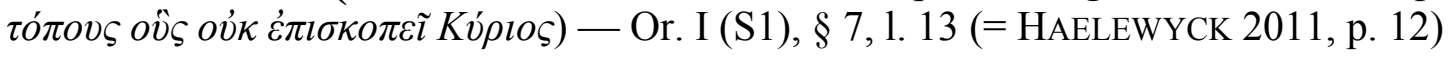

\subsubsection{PRO_pers = Les pronoms personnels}

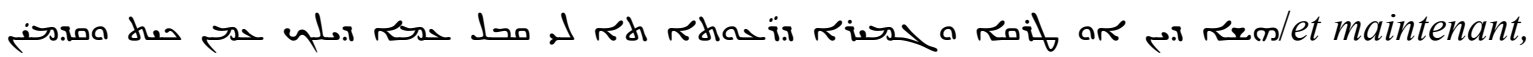
le meilleur et le plus parfait des pasteurs, venez à moi, recevez votre peuple avec nous et

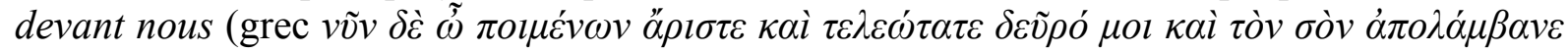

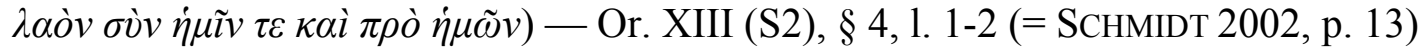

Les pronoms personnels, qu'ils soient indépendants ou suffixés, reçoivent l'étiquette

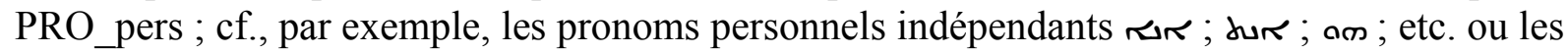

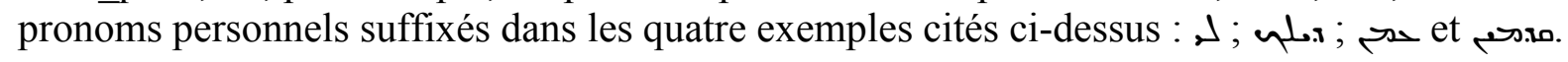

\footnotetext{
${ }^{23}$ Même si certains de ces pronoms indéfinis sont originellement des substantifs, comme لد/tout qui signifie d'abord totalité.

${ }^{24}$ Comme le signale NÖLDEKE 1898, p. 44, § 68.
} 


\subsubsection{V1-V28 = Les verbes}

Les verbes reçoivent les étiquettes V1 à V28 regroupant aussi les infinitifs et les verbes auxiliaires. Le tableau 4 fournit quelques exemples de lemmes verbaux rencontrés dans le corpus. Les analyses futures permettront certainement de développer les informations qui y sont consignées.

\begin{tabular}{|c|c|c|c|c|}
\hline Catégories & Formation & Formes & Lemmes & Racines \\
\hline V1 & Pe'al & ערתم & عרم & عרم \\
\hline V2 & Etpe'el & .xतमs & 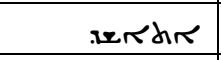 & אחR. \\
\hline $\mathrm{V} 3$ & $\mathrm{~Pa}$ "el & תלזוiזr & indis & טגi \\
\hline V4 & Etpa"al & Niiwotir & inudir & wit \\
\hline V5 & Ap'el & דוד & אח אמו. & אחכו \\
\hline V6 & Ettap'al & & & \\
\hline V7 & Šap'el & هنغكلغ & عدst & هلs \\
\hline V8 & Eštap'al & ح.roder & -radure & هis \\
\hline V9 & Sap'el & & & \\
\hline V10 & Estap'al & & & \\
\hline V11 & Tap'el & & & \\
\hline V12 & Ettap'al & & & \\
\hline V13 & Pai'el et Pai'al & रूलां & קलm & אरा \\
\hline V14 & Etpai'al & & & \\
\hline V15 & Pau'el & & & \\
\hline V16 & Etpau'al & & & \\
\hline V17 & Pal'el & & & \\
\hline V18 & Etpa'lel & & & \\
\hline V19 & Palpel & נגו.ר:ם & ح. حג. & risa \\
\hline $\mathrm{V} 20$ & Etpalpal & Dissids & pissidir & i \\
\hline V21 & Par'el & & & \\
\hline V22 & Etpar'al & & & \\
\hline V23 & Pa'li & & & \\
\hline V24 & Etpa'li & & & \\
\hline V25 & Pe'al'el & & & \\
\hline V26 & Etpe'al'el & & & \\
\hline V27 & Quadrilitères & גiv & טحi, & Rivas \\
\hline V28 & Quadrilitères passifs & passtir & pastir & riness \\
\hline
\end{tabular}

Tableau 4. Exemples de lemmes verbaux rencontrés dans le corpus

\subsection{Les racines}

L'identification de la racine pose des problèmes redoutables. Disons d'emblée que nous identifions la racine comme la base lexicale, généralement trilitère, qui a servi historiquement à former les dérivés à partir de schèmes (ou patterns) clairement reconnaissables ${ }^{25}$. Le syriaque, à l'instar des autres langues sémitiques, distingue deux catégories de noms et d'adjectifs $^{26}$ : (1) les noms et adjectifs originaux ou premiers (Primärnomen, Primär-

\footnotetext{
${ }^{25}$ Voir MOSCATI 1980, p. 76-84 ; LIPIŃSKI 2000, p. 209-228.

${ }^{26}$ KIENAST 2001, p. 59-61.
} 
substantiv, Primäradjektiv) et (2) les noms et adjectifs relevant d'une racine verbale. Dans le premier cas, c'est le nom ou l'adjectif qui est originel ; dans le second, le mot est dérivé d'une racine verbale. La plupart des noms premiers relèvent des domaines suivants: la famille (père, frère, etc.), les parties du corps (cœur, pied, etc.), les animaux (taureau, lapin, etc.), les plantes (herbe, ail, etc.), les pierres et les métaux (argile, or, etc.), les concepts temporels (année, éternité, etc.), géographiques (terre, fleuve, etc.), architecturaux (digue, toit, etc.), les ustensiles (corbeille, vase, etc.), et les mots étrangers (palais, paradis, etc.). Ils peuvent euxmêmes être à l'origine de verbes qui sont alors qualifiés de verbes dénominatifs, généralement employés à une forme dérivée. La prise en compte de ces noms premiers dispense de créer des racines verbales artificielles censées les expliquer. Les noms premiers sont ainsi classés sous un lemme et une racine identiques, toujours formulés à l'état emphatique, soit au masculin,

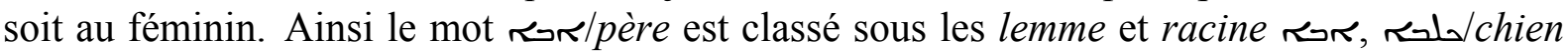

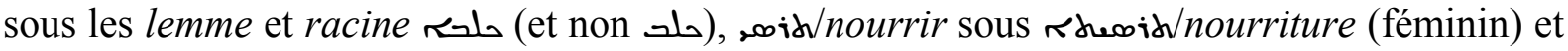
non sous une forme tap'el d'une racine ,oi, comme conjecturé par le Compendious Syriac Dictionary ou le Thesaurus Syriacus, sasdi/devenir pauvre sous / sous une forme etmap'al d'une racine 20 , comme conjecturé dans les mêmes ouvrages de consultations. Les dictionnaires, en particulier celui de M. Sokoloff, rendent ici de précieux services. Chaque entrée lexicale est suivie entre parenthèses de l'indication de la racine

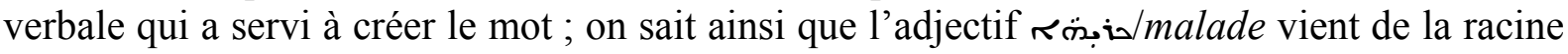
هi ; et sous sont notés tous les dérivés de cette racine. Mais, dans bon nombre de cas, le verbe est clairement identifié comme verbe dénominatif, ainsi le verbe ${ }_{5} /$ former, façonner

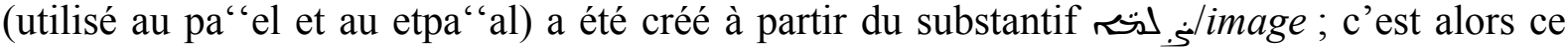
dernier qui apparaît comme racine. Cette façon de procéder, qui peut dérouter l'utilisateur, nous paraît plus respectueuse de l'histoire de la langue. L'utilisateur de la concordance en ligne devra dès lors privilégier la recherche par lemme; ce n'est que dans un second temps, quand il aura identifié la racine dont relève mot, qu'il pourra entreprendre une recherche par racine pour connaître tous les lemmes (et les formes) qui en dépendent.

Les racines homographes sont distinguées par un numéro, comme le fait M. Sokoloff. Ainsi, le lemme iuder est classé sous la racine 4 iur/rester.

Dans les concordances et les listes lexicographiques, les mots empruntés à une autre langue que le syriaque sont accompagnés du signe « (D)» (loanword, emprunt) : i $\approx<$ grec ỏń $\rho /$ air ;

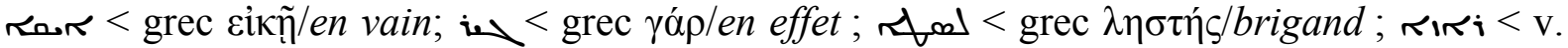
pers. *rāza-/mystère. Le tableau 5 dresse la liste des différents emprunts lexicaux rencontrés jusqu'ici dans nos données.

En cas de doute sur l'identification d'un mot comme emprunt, ou sur la langue à laquelle le mot est emprunté, nous suivons M. Sokoloff ou des ouvrages de référence faisant autorité27. C'est par exemple le cas pour la particule ה. Le Syriac Lexicon de M. Sokoloff (p. 296) se borne à évoquer l'analogie avec le grec $\delta \varepsilon ́$ quand la particule est placée en deuxième position dans un membre de phrase. Pour A. Butts, l'analogie est certes sémantique, syntaxique et phonologique, mais les deux mots doivent bien être distingués, puisque le הariaque est issu de l'araméen *'iðayn, et non du grec $\delta \dot{\varepsilon}$; le lemme syriaque n'est donc pas caractérisé ici comme un emprunt ${ }^{28}$. A contrario, le lemme i est bien considéré comme un emprunt au grec $\mu \varepsilon ́ v$ et sa racine reçoit l'indication « (L) ».

\footnotetext{
${ }^{27}$ Sur les emprunts au grec, cf. BUTTS 2016, p. 43-63; sur les emprunts aux langues iraniennes, cf. CiANCAGLINI 2008, p. 95-268.

${ }^{28}$ BUTTS 2016, p. 174-194.
} 


\begin{tabular}{|c|c|c|c|}
\hline Lemme syriaque & Langue d'origine & Lemme grec & Traduction \\
\hline ins & grec & $\dot{\alpha} \dot{\eta} \rho$ & air \\
\hline R & grec & $\varepsilon \tilde{i} \tau \alpha$ & ensuite, alors \\
\hline مRم & grec & $\varepsilon i \kappa \tilde{\eta}$ & en vain \\
\hline ארקטיה & grec & द̧́vos & étranger \\
\hline rearater & grec & $\sigma \tau i \chi \chi o \varsigma$ & stique \\
\hline 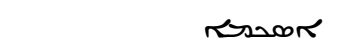 & grec & $\sigma \chi \tilde{\eta} \mu \alpha$ & forme \\
\hline Raمo.9R & grec & 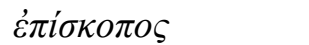 & évêque \\
\hline ie & grec & $\gamma \dot{\alpha} \rho$ & en effet \\
\hline Nam & grec & $v^{\prime \prime} \lambda \eta$ & matière \\
\hline محلي & akkadien & ekallu & temple \\
\hline (in & grec & 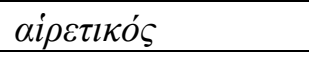 & hérétique \\
\hline Rari & akkadien & ze'pu & mensonge \\
\hline וכהז & origine discutée & & temps \\
\hline 舟借 & persan & zaina & bouclier, arme \\
\hline الس & persan & zana & manière, façon \\
\hline rogat & grec & $\tau \hat{\pi} \pi o \varsigma$ & exemple, forme \\
\hline ront & grec & $\tau \dot{x} \not \xi \zeta \varsigma$ & ordre \\
\hline reoins & akkadien & kussu & trône \\
\hline Rourafain & grec & $\chi \varepsilon \imath \rho о \tau о v \tilde{\eta} \sigma \alpha l$ & ordonner \\
\hline renativ & grec & 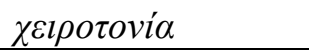 & imposition des mains \\
\hline स\&ol & grec & $\lambda \eta \sigma \tau \eta^{\prime} \varsigma$ & bandit \\
\hline تخحقمك & akkadien & makisu & collecteur d'impôt \\
\hline ن & grec & $\mu \varepsilon^{\prime} v$ & d'une part \\
\hline revers & akkadien & muškenu & pauvre \\
\hline roonss & grec & vómos & loi \\
\hline stroo & persan & asem & argent (métal) \\
\hline roing & grec & $\pi o ́ \rho o \varsigma$ & moyen, façon \\
\hline No.9.9 & grec & $\pi \varepsilon \tilde{l} \sigma l \varsigma$ & persuasion \\
\hline & hébreu & panîm & tourner \\
\hline $\mathrm{Ru}_{5} \mathrm{~g}$ & hébreu & pèsaḥ & Pâques \\
\hline Rifooig & grec & $\pi \rho o \sigma \tau \dot{\alpha} \varsigma,-\dot{\alpha} \delta \alpha$ & portique \\
\hline Sod do & persan & patigama & mot, phrase \\
\hline مصلهم & grec & $\kappa \alpha ́ \lambda \omega \varsigma$ & louange \\
\hline مسمس & grec & $\kappa \alpha v \omega ́ v$ & canon, loi \\
\hline riri & persan & $r a z a$ & secret, mystère \\
\hline रivd & akkadien & tamkaru & commerçant \\
\hline rengh & origine discutée & & prototype \\
\hline
\end{tabular}

Tableau 5. Les emprunts rencontrés dans le corpus

\section{3. Édition de concordances et de listes lexicographiques}

À l'issue des traitements, les textes et les informations de lemmatisation qui les accompagnent sont réunis dans des bases de données ${ }^{29}$. Des outils spécifiques, développés par

${ }^{29}$ En l'occurrence, le système de gestion de base de données Microsoft SQL Server. 
les informaticiens du CENTAL, permettent d'extraire de ces bases de données les informations nécessaires pour répondre à une requête ${ }^{30}$. Ces informations sont organisées et affichées selon des mises en pages prédéfinies permettant de les visualiser dans les outils lexicographiques, à savoir des concordances, des index ou des listes alphabétiques ou fréquentielles. Les requêtes peuvent porter sur l'ensemble du texte ou sur une portion du texte. Elles peuvent aussi concerner un ou plusieurs éléments des données lemmatisées, une forme, un lemme, une catégorie morphosyntaxique, une racine.

Les pages qui suivent illustrent ces différents types de sortie, ici au format ADOBE PDF : une concordance grecque (figure 4), une concordance syriaque (figure 5), deux concordances bilingues grec-syriaque (figures 6 et 7), une liste bilingue grec-syriaque des emplois du lemme $\theta \varepsilon$ ó (figure 8), une liste bilingue syriaque-grec du lemme concordance bilingue syriaque-grec des lemmes caractérisés comme noms propres (figure 10).

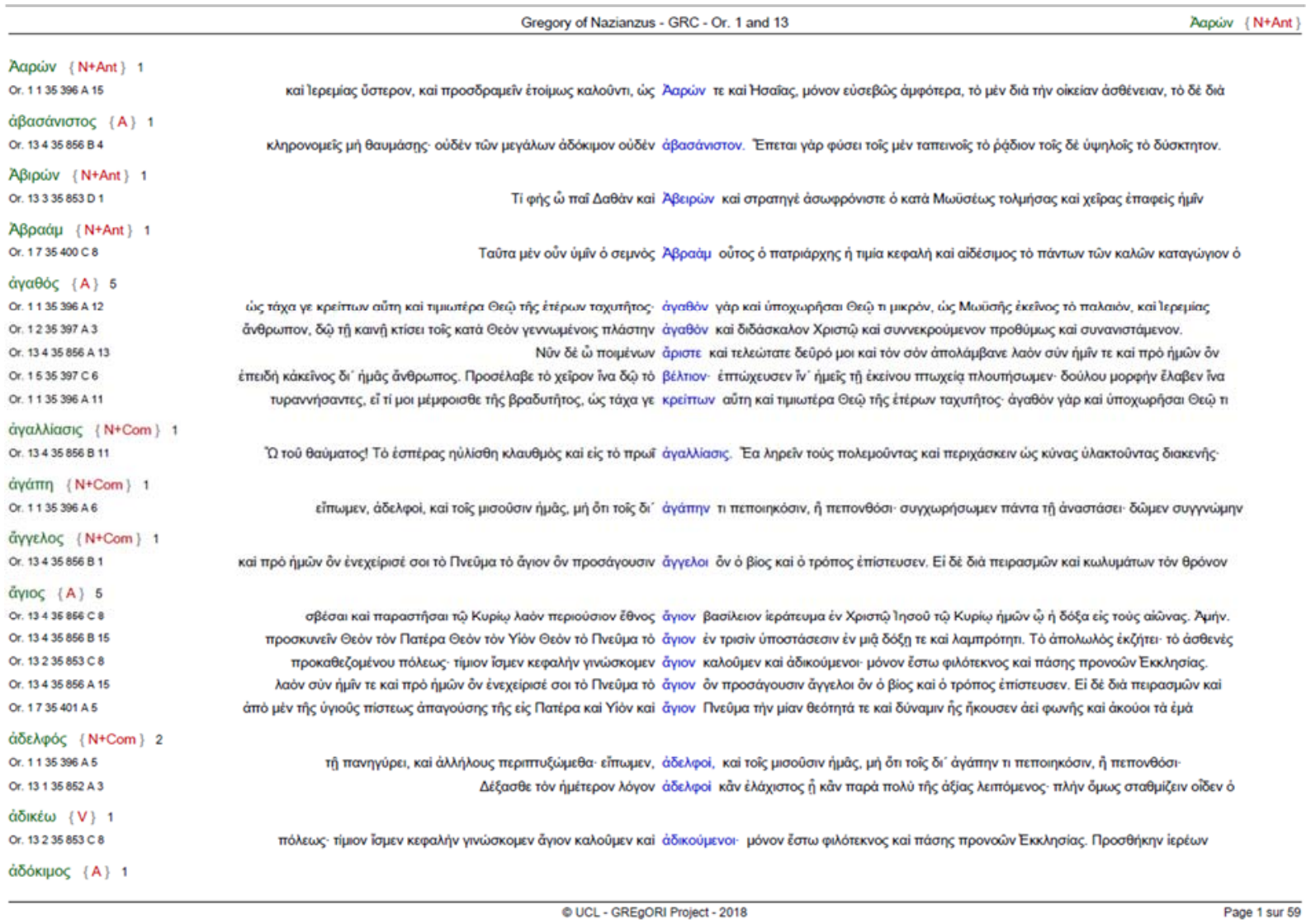

Figure 4. Première page de la concordance lemmatisée du corpus grec

\footnotetext{
${ }^{30}$ Ces outils utilisent, en l'occurrence, SAP Crystal Reports, un logiciel de représentation graphique de données.
} 


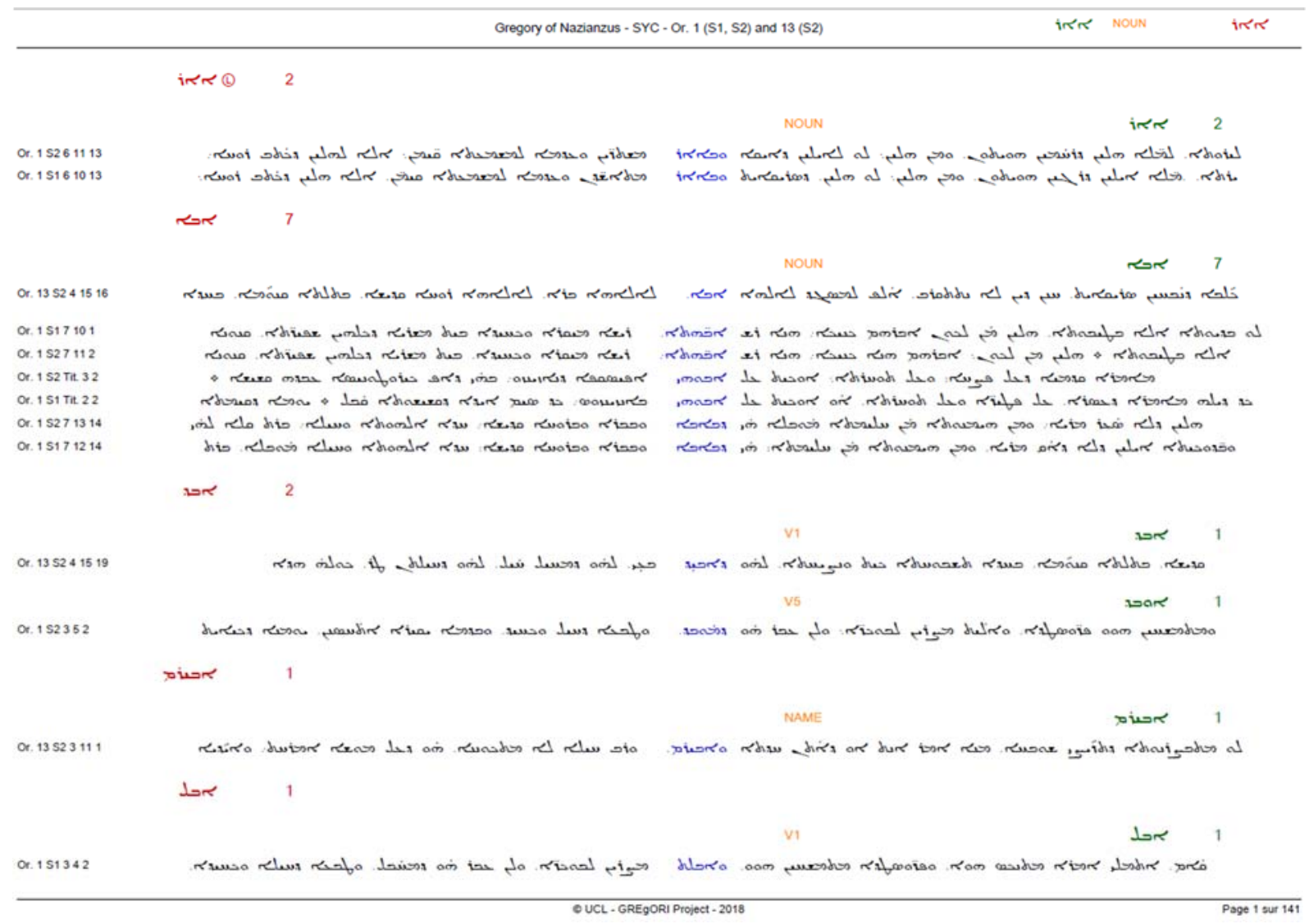

Figure 5. Première page de la concordance lemmatisée du corpus syriaque

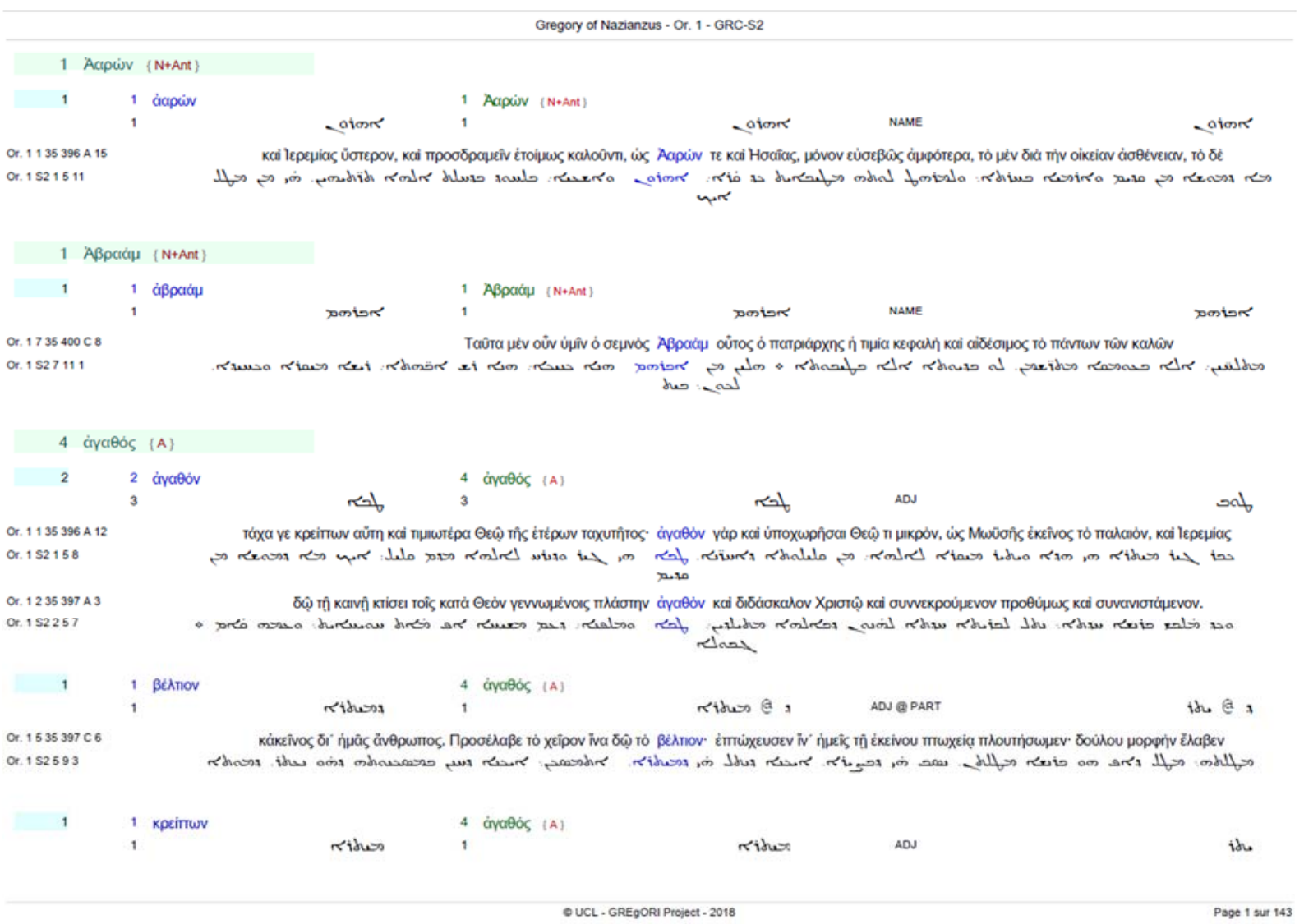

Figure 6. Première page de la concordance lemmatisée grec-syriaque (S2) du Discours 1 


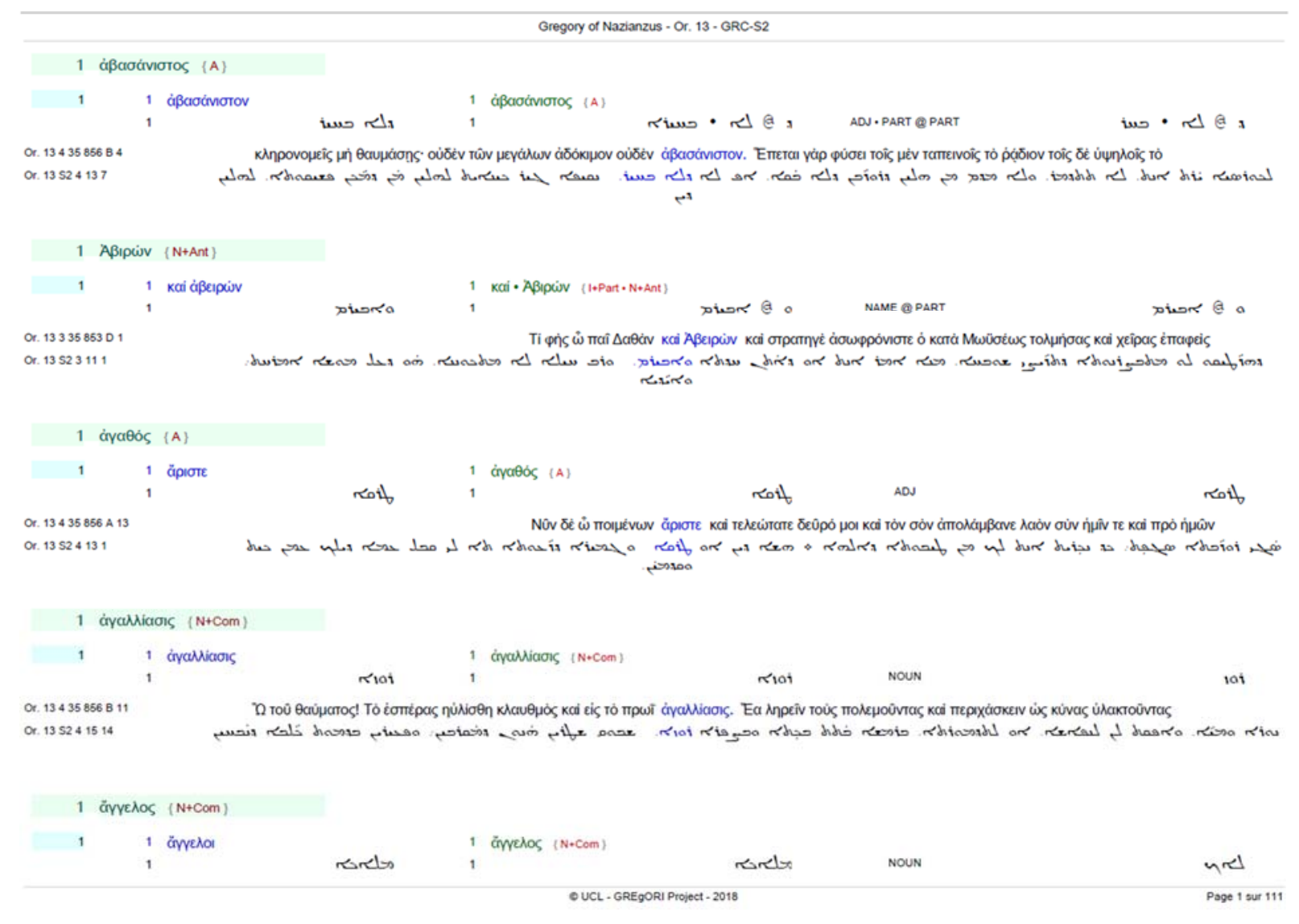

Figure 7. Première page de la concordance lemmatisée grec-syriaque (S2) du Discours 13

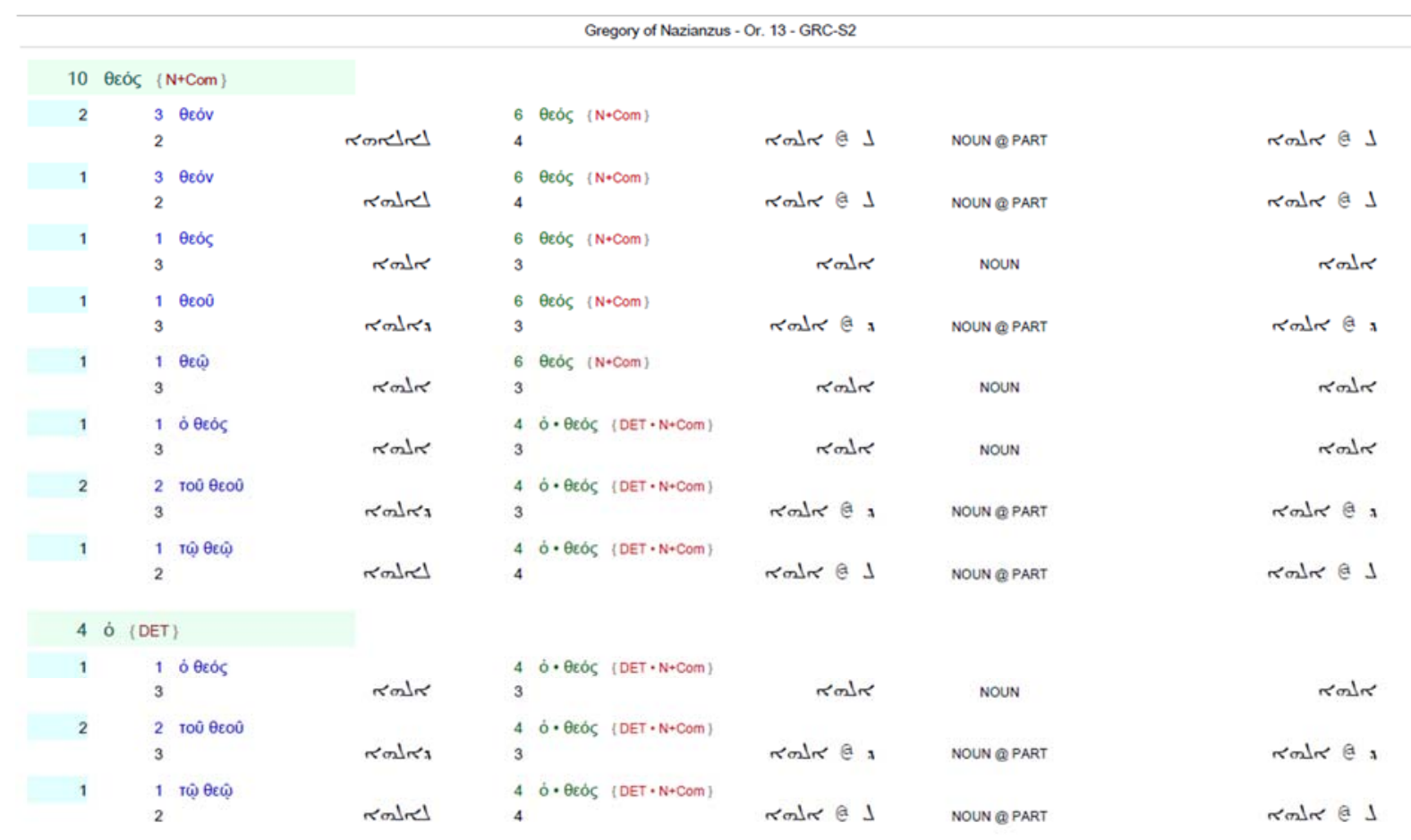




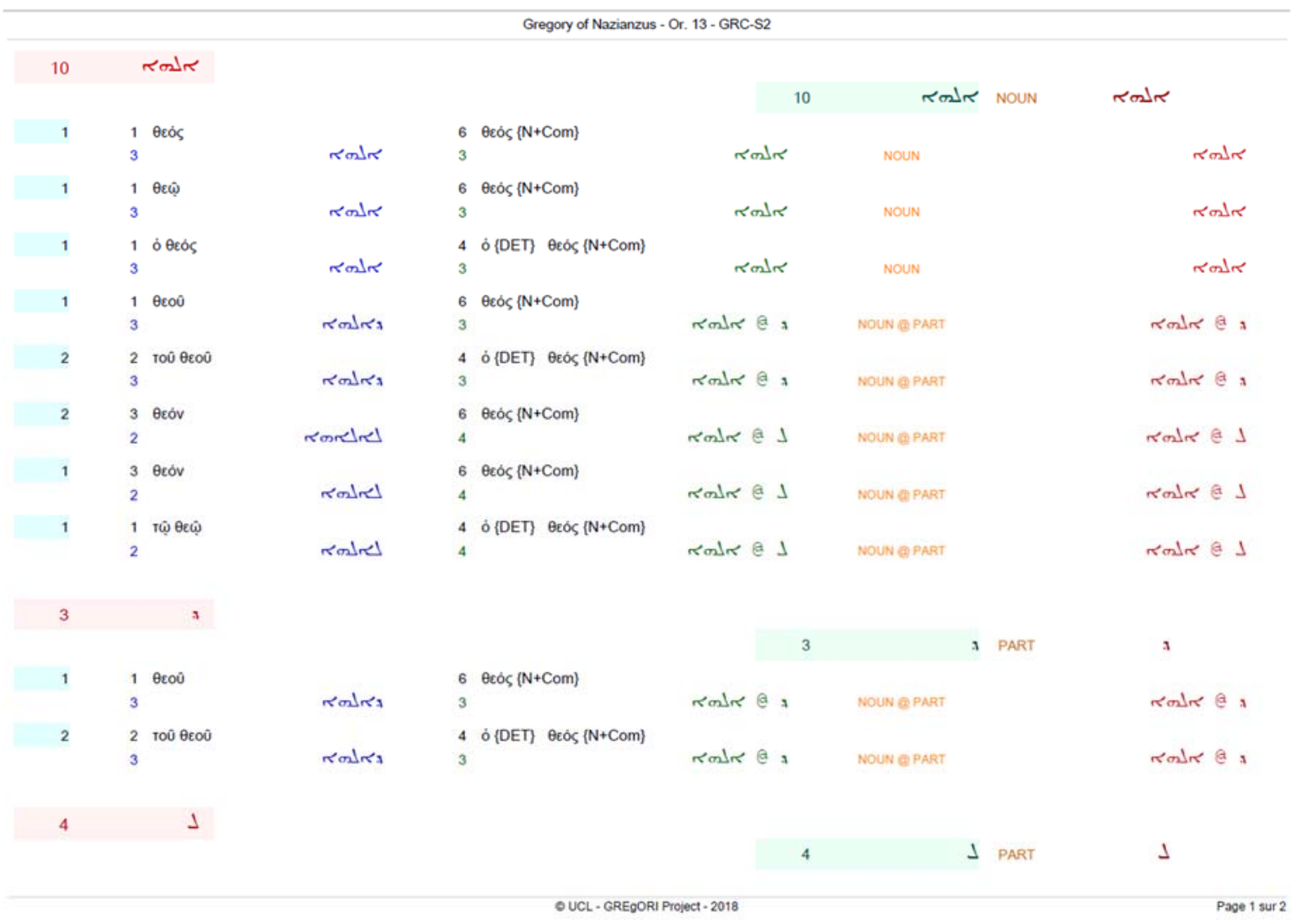

Figure 9. Liste bilingue syriaque (S2)-grec du lemme rod dans le Discours 13

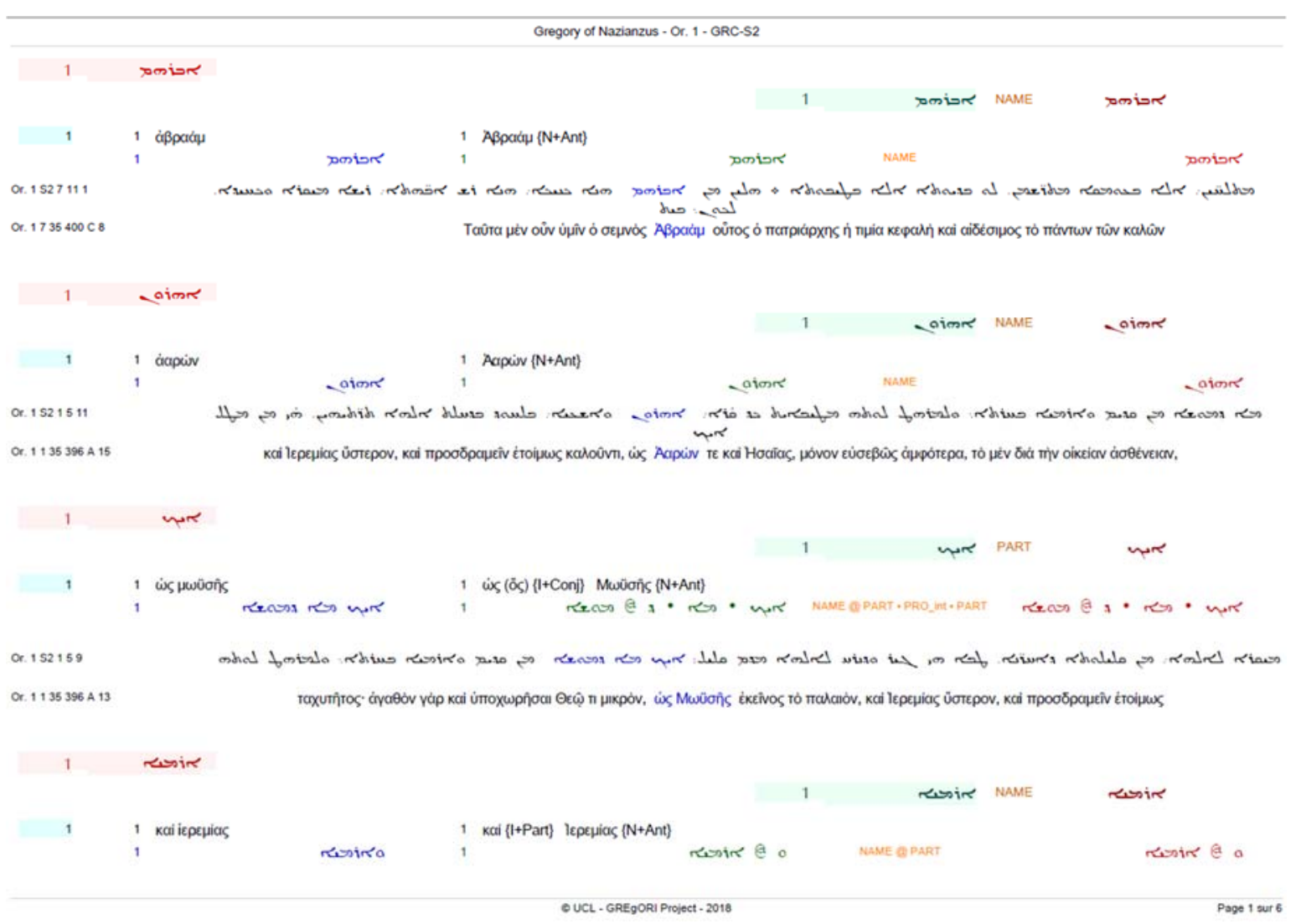

Figure 10. Extrait de la concordance bilingue syriaque (S2)-grec des lemmes catégorisés comme noms propres (NAME) dans le Discours 13 


\section{L'interface en ligne}

L'interface en ligne, déjà opérationnelle dans une version expérimentale, permet d'interroger les corpus sur un ordinateur personnel. Nous nous limitons ici à décrire l'interface permettant d'interroger le texte grec et la version syriaque (S1) du Discours 1. Visuellement, cette interface est volontairement simple. Un premier registre identifie, à gauche, l'auteur (Grégoire de Nazianze) et le texte interrogés (le Discours 1). Il présente aussi, à droite, deux boutons: (1) une flèche vers le bas inscrite dans un cercle (figure 11) propose un menu permettant de télécharger des concordances et des outils lexicographiques - document décrits dans la Section 3, ci-dessus - au format ADOBE PDF et (2) la lettre « $\mathrm{i}$ » inscrite dans un cercle (figure 12), ouvre une fenêtre contextuelle (pop-up window) indiquant les références bibliographiques précises des éditions suivies (le texte de base), identifiant les personnes responsables de la lemmatisation et de l'alignement des textes, et signalant les institutions impliquées dans le traitement du corpus en question.

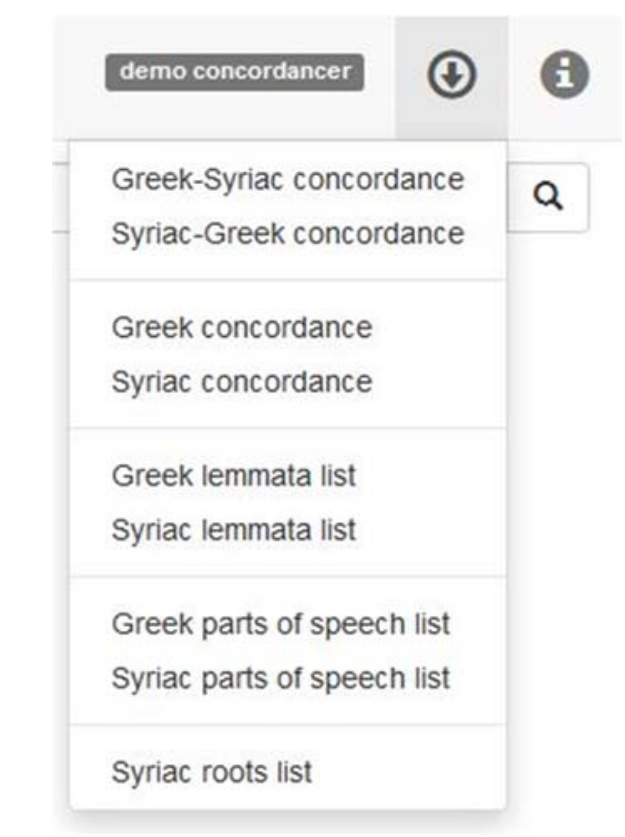

About

Demo concordancer

Software and linguistic data are subject to improvements or corrections. We apologize in advance for any defects or errors that users may still come across.

Edition

Greek text : Patrologia Graeca, 35, col. 395-402

Syriac version (\$2) : Sancti Gregoril Nazianzeni Opera. Versio Syriaca V. Orationes 1, II, III (Corpus Christianorum. Series Graeca, 77. Corpus Nazianzenum, 25), ed. J-C.

Haelewyck, Turnhout, 2011 (Versio Recentio [S1], p. 3-13)

Lemmatisation

Greek: Bernard COULE, Bastien KINDT, Justin MOSSAY†

Syriac: Jean-Claude HAELEWYCK, Bastien KINDT, Andrea SCHMIDT

Alignment

Jean-Claude HAELEWYCK, Bastien KINDT

Technical developments

Hubert NAETS, Claude DEVIS

CENTAL (https S//mww uclouvain be/cental htmil)

GREgORI Project (nttp://mwruclouvain. be/gregori-project)
Figure 11. Affichage de la liste des documents téléchargeable au format $A D O B E P D F$

Figure 12. La fenêtre contextuelle décrivant le corpus exploré 
Le second registre constitue le champ d'interrogation. À gauche, un menu permet de sélectionner la langue interrogée, le grec du texte source ou le syriaque du texte cible (ici, S1). La

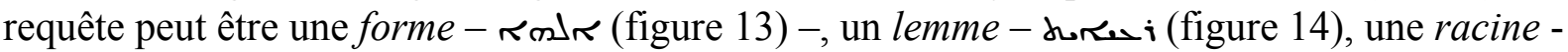
- ${ }_{\text {- }}$ (figure 15) -, une catégorie morphosyntaxique - par exemple les verbes Etpa “al (V4) (figure 16) - ou une combinaison de ces trois éléments - par exemple les séquences constituées d'une forme d'un lemme verbal Pe'al («V4») suivi d'une forme relevant de la racine, i (figure 17). Les occurrences du texte syriaque sont analysées en tenant compte des éléments préfixés et suffixés. Il est possible de formuler une requête cherchant toutes les occurrences constituées des particules a et $\rfloor$ suivies d'un lemme catégorisé comme nom. Cette recherche fournit la concordance des formes alda, (figure 18).

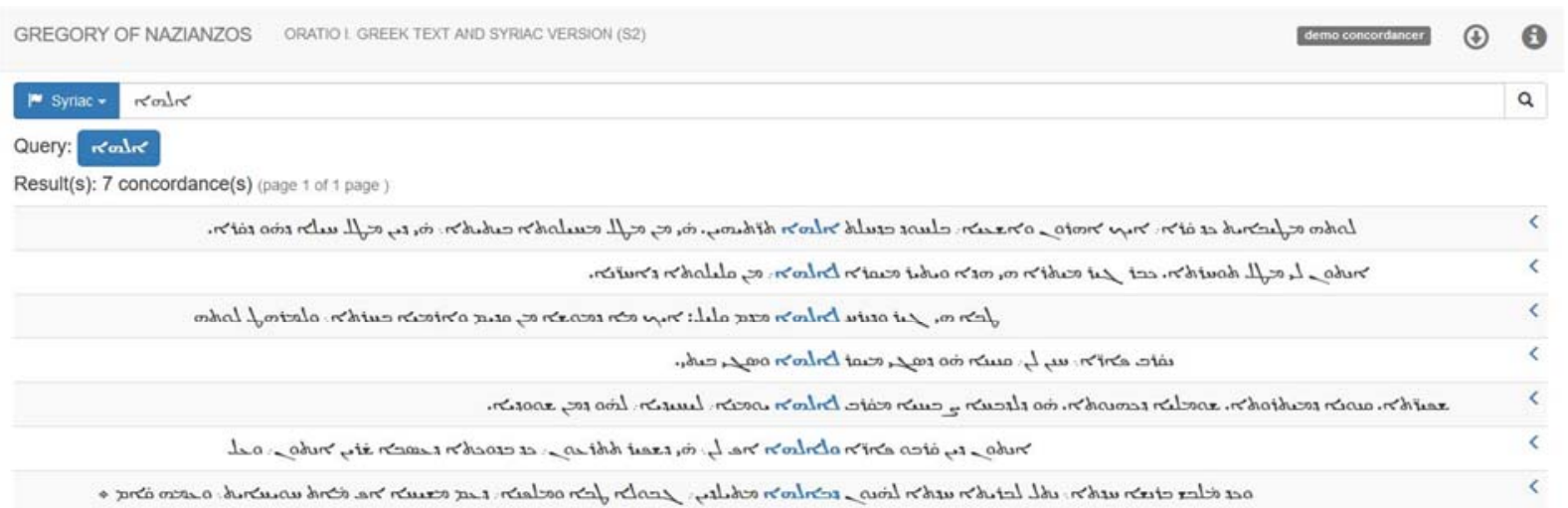

Figure 13. Recherche des occurrences de la forme rod 


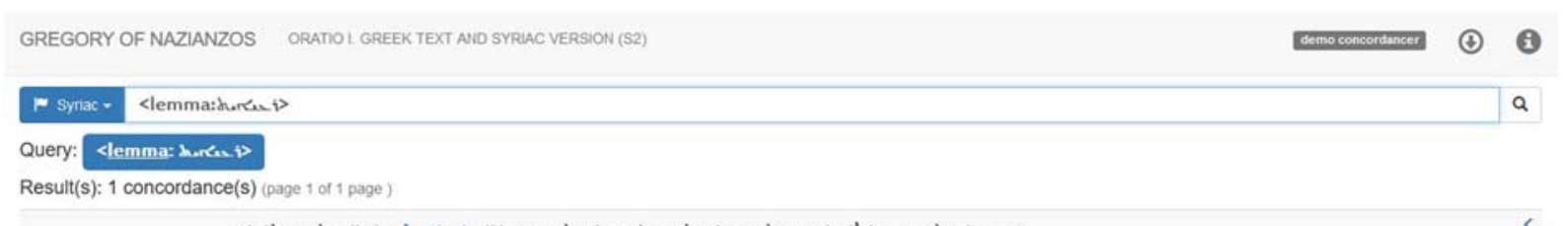

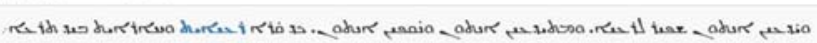

CENTAL)

02017 Centre de trattement automatique du langage (Cental) / GREgoRI Project (Universtéte catholque de Louvain)

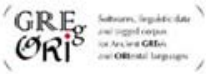

Figure 14. Recherche des occurrences du lemme durai

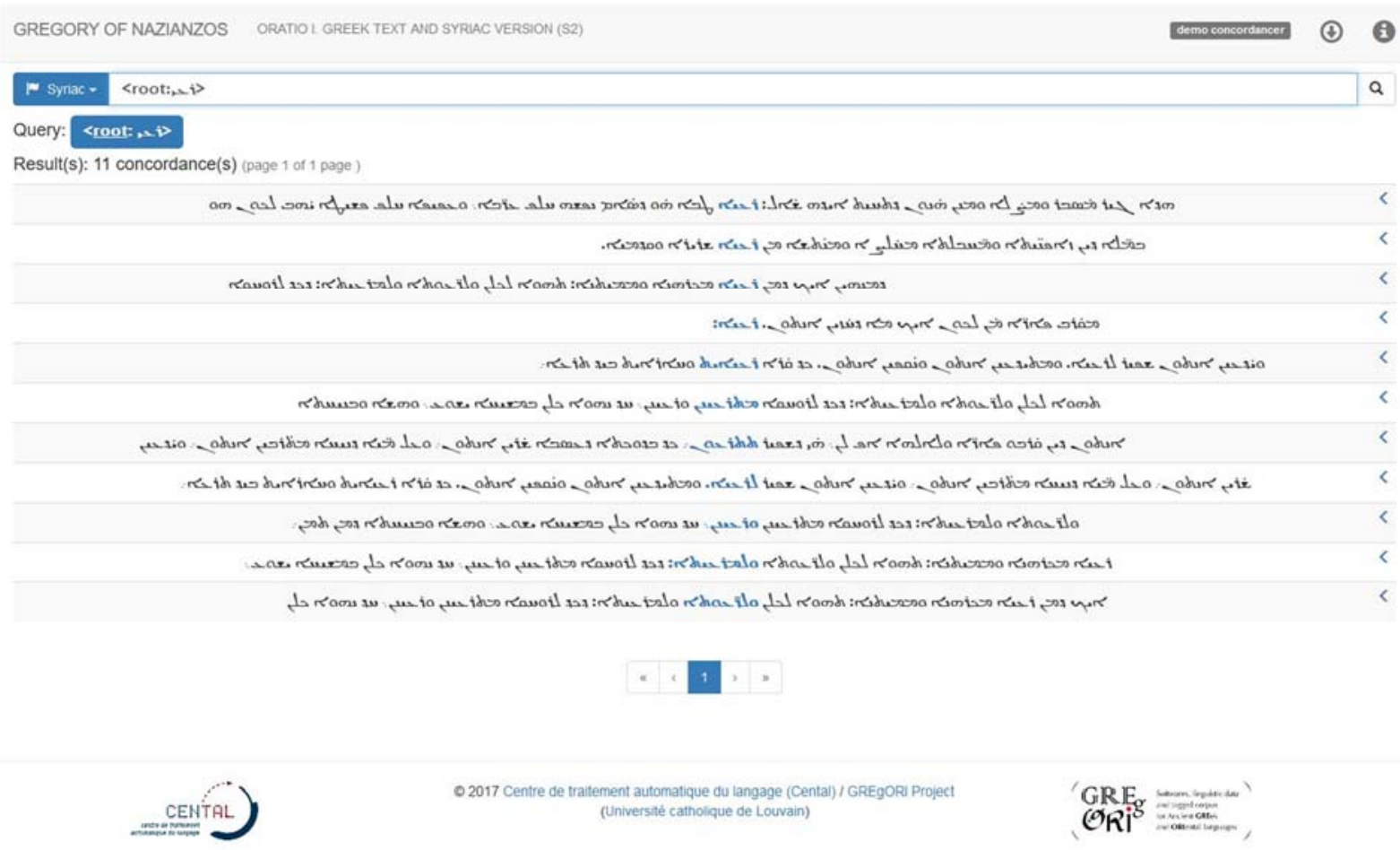

Figure 15. Recherche des occurrences de la racine, $\mathrm{i}$ 


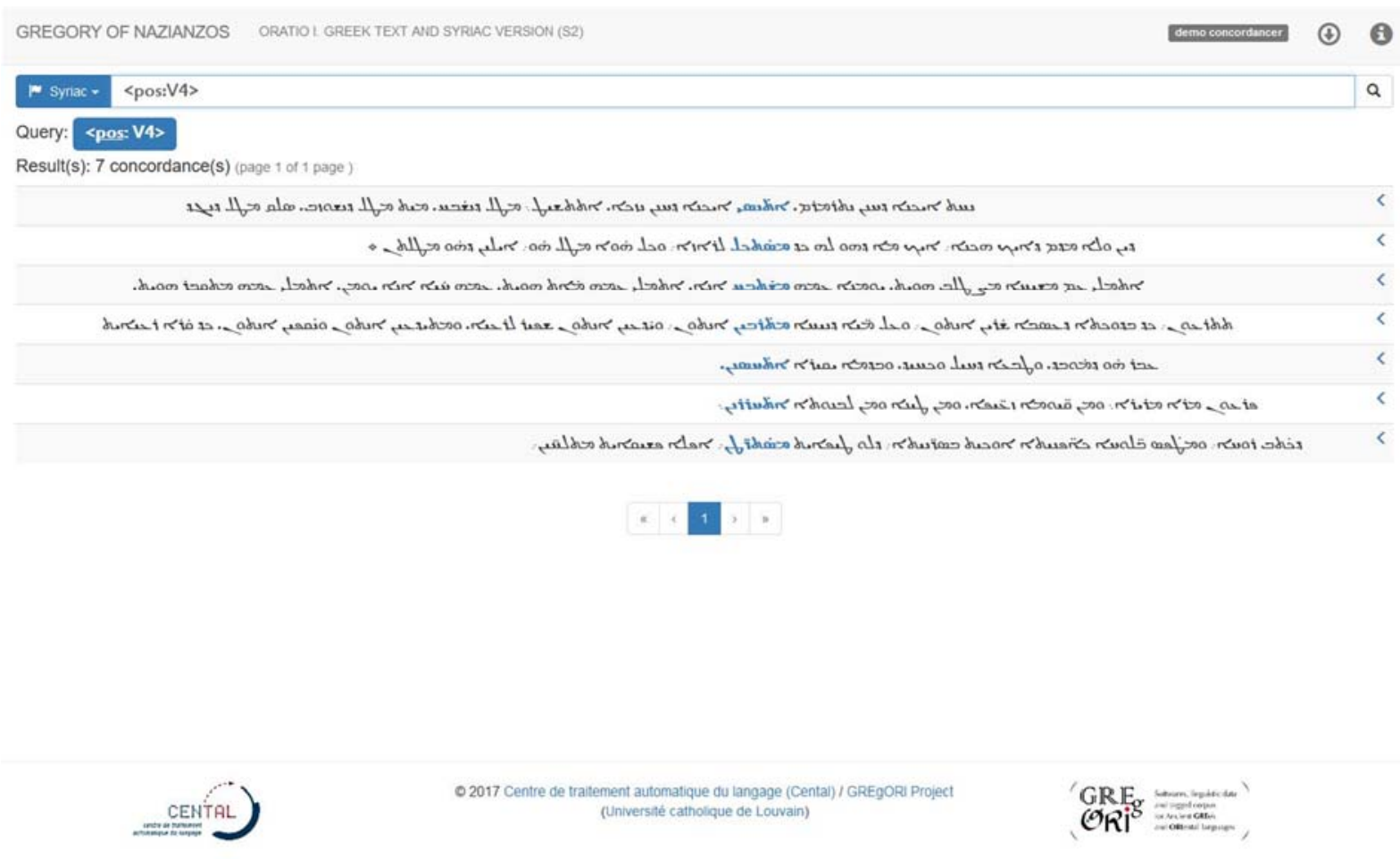

Figure 16. Recherche des occurrences des formes relevant de lemmes verbaux Etpa "al (étiquette «V4»)

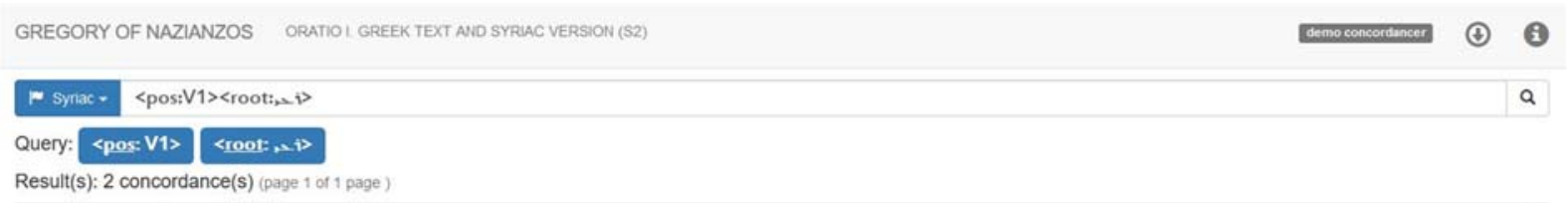

.

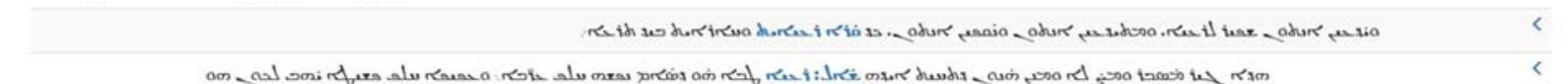

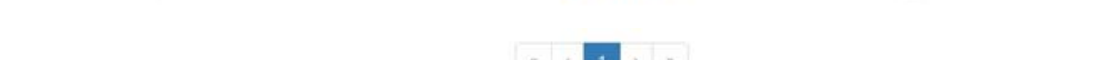



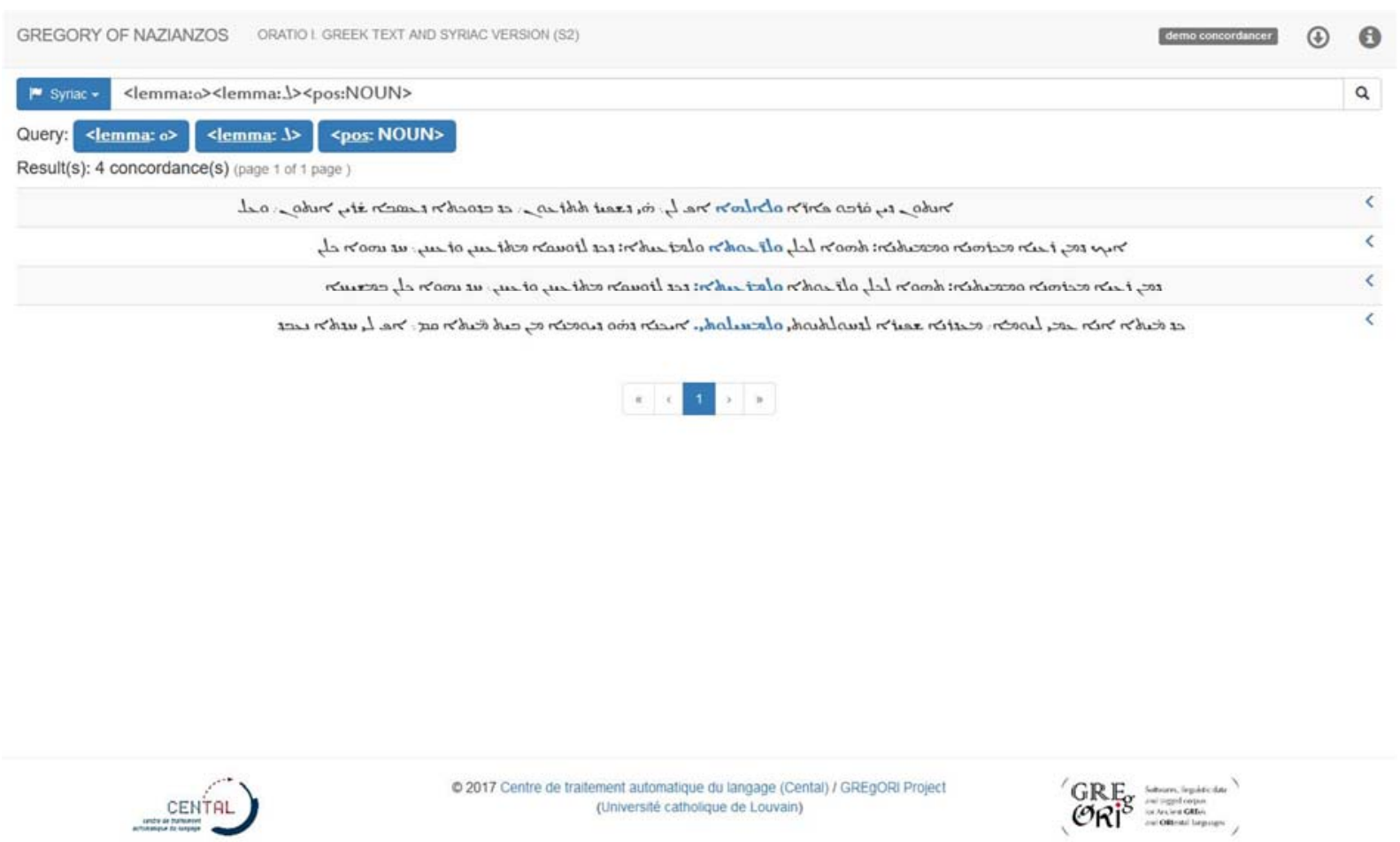

02017 Centre de tratement automatique du langage (Cental) / GREgoru Project (Universtét cathoique de Louvain)

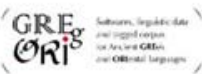

Figure 18. Recherche des occurrences des formes

constituées des préfixes o et $\lrcorner$ et d'une forme relevant d'un lemme nominal

Dans son état actuel, l'utilisation de l'interface exige d'utiliser des balises spéciales pour interroger les lemmes - «<lemma:[UN LEMME]> » -, les catégories morphosyntaxiques «<pos:[UNE CATÉGORIE MORPHOSYNTAXIQUE] («pos» signifiant Part-ofSpeech/partie du discours) -, et les racines - «<root:[UNE RACINE]> ». À l'avenir, un menu d'aide permettra à l'utilisateur de concevoir ses requêtes de manière plus intuitive. Les requêtes sont systématiquement récapitulées sous le champ d'interrogation, après la mention «QUERY », comme illustré sous la figure 18.

Les résultats de ces requêtes s'affichent sous la forme d'une concordance. Chaque ligne de cette concordance donne accès, en cliquant sur la flèche située à droite (pour les langues qui s'écrivent de droite à gauche) ou à gauche (pour les langues qui s'écrivent de gauche à droite), à un contexte étendu. Ce dernier est plus large que celui de la ligne de la concordance et offre le texte grec et sa traduction syriaque (figure 19). Il est accompagné des références précises de la forme dans les textes de base. En positionnant le curseur sur une forme du texte source ou du texte cible, l'analyse de cette forme (lemme et catégorie morphosyntaxique) apparaît et les équivalences de traduction dans les deux textes, mots ou expressions, sont mises en surbrillance. 


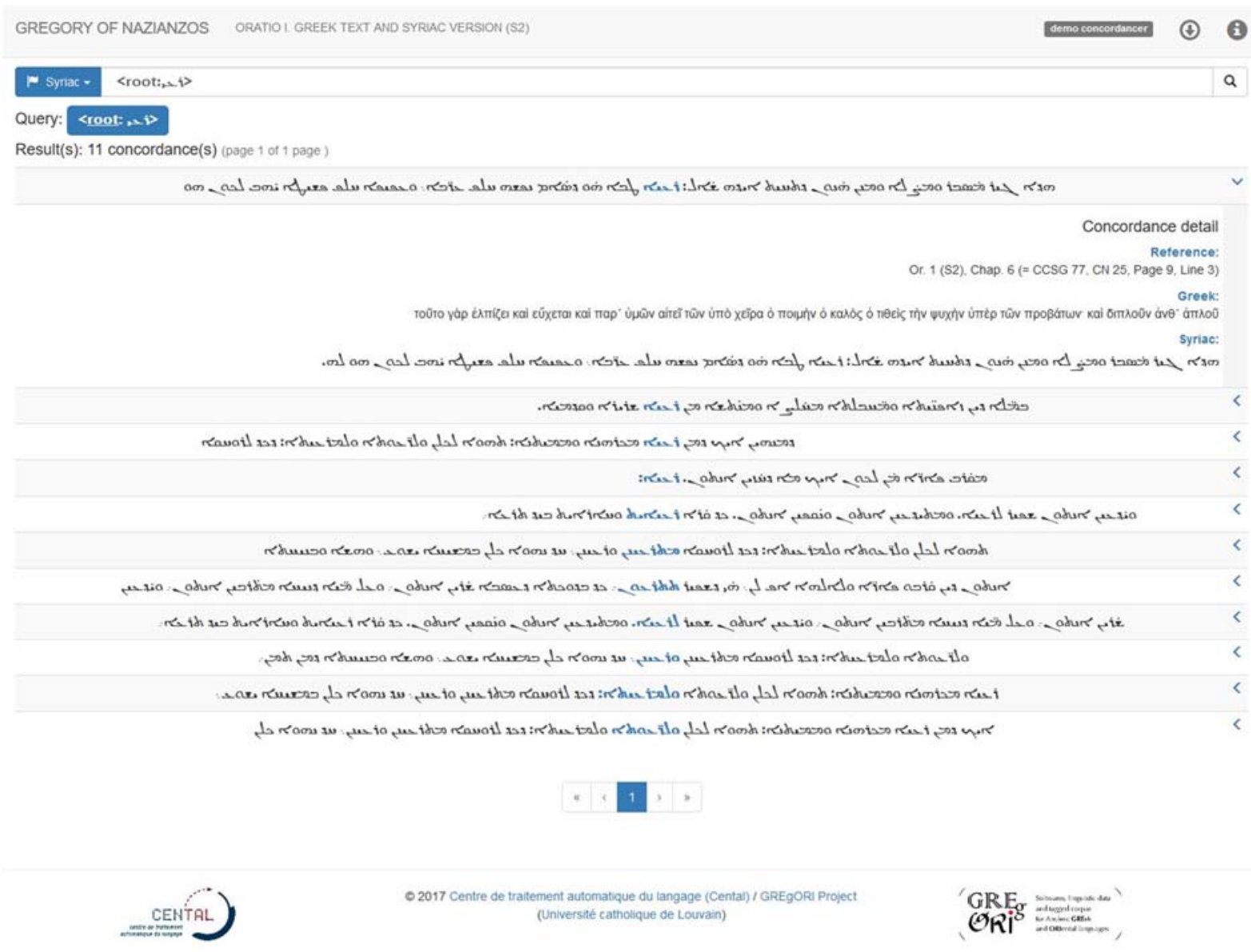

Figure 19. Affichage du contexte étendu ("Concondance details ») d'une occurrence

\section{Conclusions et perspectives}

D'autres textes sont d'ores et déjà en cours de traitement. A.B. Schmidt et G. Abousamra travaillent à l'édition et à l'analyse lexicale de textes magiques. J.-Cl. Haelewyck et B. Kindt assurent le traitement des trois versions syriaques de l'Historia Zosimi de Vita Beatorum Rechabitarum. B. Kindt et N. Atas ont préparé une concordance bilingue (syriaque - anglais) de l'Oratio Manasseh, sur base de données lexicales produites par le Peshitta Institute - Eep Talstra Centre for Bible and Computer (Amsterdam). N. Atas a analysé le lexique des chapitres I-X de la Genèse, en syriaque et dans leur version en turoyo ${ }^{31}$. A.S. Sembiante, J.$\mathrm{Cl}$. Haelewyck et $\mathrm{B}$. Kindt ont lemmatisé la version syriaque du premier poème dogmatique de Grégoire de Nazianze; Y. Arzhanov collabore avec A.B. Schmidt et B. Kindt pour produire la lemmatisation de la version syriaque des Sentences des philosophes grecs, un texte qui paraîtra dans le Corpus Scriptorum Christianorum Orientalium.

Ces travaux permettront d'éprouver les principes d'analyse présentés dans cet article et contribueront à enrichir quantitativement et qualitativement les corpus et les données linguistiques. Ils serviront aussi à constituer, progressivement, un dictionnaire de lemmatisation dédié à l'analyse de la langue syriaque, comme cela existe déjà pour le grec et d'autres langues de l'Orient chrétien.

\footnotetext{
${ }^{31}$ Ce travail a jeté les bases du traitement automatique du turoyo, cf. ATAS 2018. Les concordances syriaque et turoyo de Gen. I-X sont mises à la disposition des chercheurs sur le site du projet GREgORI.
} 
Les interfaces accessibles en ligne offrent un accès aux textes par l'intermédiaire des concordances. Les éléments présents dans ces concordances correspondent à des questions spécifiques posées par l'utilisateur. Ces questions, appelées « requêtes », peuvent porter sur les racines (dans le cas des langues sémitiques), sur les lemmes, sur les formes, sur les catégories grammaticales, c'est-à-dire sur toutes les informations linguistiques attachées aux mots présents dans le corpus exploré ${ }^{32}$. Les requêtes peuvent également être constituées d'une combinaison de ces différents éléments. Une fois la requête lancée, la concordance s'affiche instantanément à l'écran.

Ces concordances sont dites «dynamiques ». Les concordances imprimées sur papier, et diffusées sous forme de livres, sont quant à elle des concordances dites "statiques ». Ces concordances-livres, qui existent depuis longtemps, tant en grec qu'en syriaque, seront amenées à être remplacées par d'autres outils, tels que ceux décrits dans ces lignes. Bien entendu, ces ouvrages ont rendu d'importants services aux chercheurs ${ }^{33}$. Mais leur diffusion sur papier (ou, dans certains cas, sur microfiches) constitue une contrainte matérielle importante. Cette contrainte interdit de corriger les données ou de les adapter, d'une part, et offre peu de possibilités de recherche, d'autre part. Les limites physiques de la page du livre obligent les auteurs et les éditeurs à limiter drastiquement la longueur des contextes. Le pivot, le mot-clé théoriquement encadré de contextes en amont et en aval, est systématiquement une forme de mot et jamais une expression. Il est impossible de faire varier le tri des racines, des lemmes, des formes et des contextes en fonction de l'ordre alphabétique ou de l'ordre de leur apparition dans les textes, selon les besoins de l'utilisateur. Enfin, au niveau théorique, chaque publication repose sur des règles de dépouillement et d'analyse qui lui sont propres ${ }^{34}$ et, à quelques rares exceptions près ${ }^{35}$, ces principes demeurent largement implicites ${ }^{36}$.

Les efforts en matière d'alignement des textes sources sur les différents textes cibles disponibles ouvrent des perspectives nouvelles. L'automatisation des processus d'analyse permet

${ }^{32}$ Il est évidemment possible d'enrichir davantage la nature et le nombre des analyses linguistiques proposées. En grec, par exemple, certains développements utilisent les analyses flexionnelles (cas, genre, nombre, voix, mode, temps, personne, etc.), mais, de manière générale, les travaux du projet GREgORI s'arrêtent volontairement au niveau de l'analyse lexicale.

${ }^{33}$ Sans prétendre ici à l'exhaustivité, on peut citer, pour le grec, la concordance de la Septante réalisée par E. Hatch et H.A. Redpath (première édition 1897), ouvrage qui a longtemps fait figure de modèle. Pour les auteurs classiques, il faut mentionner, à titre d'exemple, les concordances publiées par la maison d'édition OlmsWeidmann dans la collection des Lexika, Indizes, Konkordanzen zur Klassischen Philologie (cf. http://www.olms.de/search/result.aspx?SeriesID=002). Pour les sources patristiques et byzantines, il faut épingler les volumes du Thesaurus Patrum Graecorum, une collection de concordances lemmatisées, jadis produites par les promoteurs du projet GREgORI et diffusées par Brepols Publishers (voir la liste des titres parus sur le site du projet, cité note 3 ; dans ce dernier cas, les concordances étaient publiées sur des microfiches). En syriaque, on se doit de citer les ouvrages de AUfreCHT, Hurd 1975 ; KIRAZ 1993 ; WinTER 1976 ; BORBONE et alii 1997 ; LUND, KIRAZ 2004.

${ }^{34}$ Dans la collection des Lexika, Indizes, Konkordanzen, diffusée par Olms-Weidmann, le même lexème

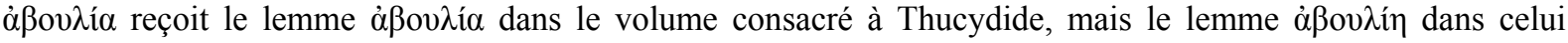
consacré à Hérodote. C'est donc la langue des auteurs qui est décrite (ionio-attique pour le premier, ionienne pour le second) et non la langue grecque en général ; sur cette distinction, cf. KINDT 2004, p. 224, §6. Dans le cadre du projet GREgORI, l'analyse se situe toujours au niveau de la description générale de la langue.

${ }^{35}$ Voir, par exemple, la concordance du Pentateuque dans la Peshițta publiée par P.G. Borbone, J. Cook, K.D. Jenner et D.M. Walter.

${ }^{36} \mathrm{La}$ Concordance to the Syriac New Testament de G.A. Kiraz commence ex abrupto, sans aucune explication relative aux textes suivis ou aux principes d'analyse adoptés. 
de travailler sur de vastes corpus. Elle procure des résultats tangibles, susceptibles d'être utiles aux recherches dans différents domaines comme l'étude des méthodes de traduction ou la linguistique contrastive, par exemple.

L'accès en ligne permet d'offrir aux chercheurs un corpus destiné à s'accroître au fil du temps. Le Digital Syriac Corpus (DSC) vient d'ouvrir la voie en ce domaine ${ }^{37}$. Les développements réalisés dans le cadre du projet GREgORI s'inscrivent dans la même démarche, mais en tâchant de répondre à trois exigences supplémentaires: (1) les textes sont munis d'un étiquetage lexical (lemmes et racines) et morphosyntaxique (les catégories) abouti ; (2) le résultat de l'interrogation des textes s'affiche sous la forme de concordances à partir desquelles l'utilisateur peut accéder aux textes ; (3) les textes sources sont alignés sur leurs textes cibles.

L'expérience acquise dans le domaine de l'analyse du syriaque permet aux promoteurs du projet GREgORI, et à leurs collaborateurs, de s'atteller désormais à l'analyse de deux ensembles textuels majeurs, la version syriaque des homélies de Grégoire de Nazianze publiée dans le Corpus Nazianzenum, une sous-série du Corpus Christianorum publié par Brepols Publishers (103.305 occurrences), et celui des œuvres d'Éphrem de Nisibe publiées par E. Beck dans les volumes du CSCO (301.623 occurrences).

\footnotetext{
${ }^{37} \mathrm{Cf}$. https://syriaccorpus.org.
} 


\section{BIBLIOGRAPHIE}

Anonyme 1985: The concordance to the Peshitta version of the aramaic New Testament, New Knoxville.

Arzhanov 2018 : Y. Arzhanov, Syriac Sayings of Greek Philosophers. A Study in Syriac Gnomologia. With Edition and Translation, Leuven (à paraître dans la série des Subsidia du Corpus Scriptorum Orientalium Christianorum).

ATAS 2018 : N. ATAS, Édition et étude philologique du manuscrit turoyo Sachau 249 de la Staatsbibliothek de Berlin, Mémoire de Master en langues et lettres anciennes, orientation orientales, Université catholique de Louvain, Louvain-la-Neuve, 2017-2018 (inédit).

Aufrecht, Hurd 1975 : W.E. Aufrecht, J.C. Hurd, A Synoptic Concordance of Aramaic Inscriptions according to H. Donner and $W$. Röllig (The International Concordance Library, 1), Missoula.

Borbone et alii 1997 : P.G. Borbone, J. COOK, K.D. JENnER, D.M. WALter, in collaboration with J.A. Lund, M.P. WeItzMan, The Pentateuch (The Old Testament in Syriac according to the Peshitta. Part V. Concordance. Volume 1), Leiden, New York, Cologne.

ButTs 2016 : A.M. ButTS, Language Change in the Wake of Empire. Syriac in Its GrecoRoman Context (Linguistic Studies in Ancient West Semitic, 11), Winona Lake.

CiancAglini 2008 : C.A. CianCAGLini, Iranian Loanwords in Syriac (Beiträge zur Iranistik, 28), Wiesbaden.

Costaz 2002 : L. Costaz, Dictionnaire Syriaque-Français. Syriac-English Dictionary, $3^{\mathrm{e}}$ ed., Beyrouth.

Coulie 1996 : B. Coulie, «La lemmatisation des textes grecs et byzantins : une approche particulière de la langue et des auteurs », Byzantion, 66, p. 35-54.

Coulie et alii 2013 : B. Coulie, B. Kindt, T. PATARIDZE, " Lemmatisation automatique des sources en géorgien ancien », Le Muséon, 126, p. 161-201.

Duval 1881 : R. Duval, Traité de grammaire syriaque, Paris (réimpr. Piscataway, 2010).

FLEURY 2012 : S. FleURY, mkAlign, Manuel d'utilisation, Université Sorbonne Nouvelle Paris 3 [pdf].

HaElewyck 1998 : J.-Cl. HaelewyCK, Clavis Apocryphorum Veteris Testamenti (Corpus Christianorum), Turnhout.

HAELEWyCK 2011 : J.-Cl. HAELEWYCK, Sancti Gregorii Nazianzeni Opera. Versio Syriaca. V. Orationes I, II, III (Corpus Christianorum. Series Graeca, 77 ; Corpus Nazianzenum, 25), Turnhout.

Haelewyck 2014 : J.-Cl. Haelewyck, « Historia Zosimi de Vita Beatorum Rechabitarum. Édition de la version syriaque brève », Le Muséon, 127, p. 95-147.

HAELEWYCK 2015 : J.-Cl. HAELEWYCK, « La version syriaque longue de l'Historia Zosimi de Vita Beatorum Rechabitarum. Édition et traduction », Le Muséon, 128, p. 295-379.

HAELEWYCK 2017a : J.-Cl. HAELEWYCK, «Histoire de Zosime sur la vie des Bienheureux Réchabites. Les trois recensions syriaques. Édition de la version résumée », Parole de l'Orient, 43, p. 175-194. 
HAELEWYCK 2017b : J.-Cl. HAELEWYCK, «Les versions syriaques des Discours de Grégoire de Nazianze : un processus continu de révision », BABELAO, 6, p. 75-124 [pdf].

Hatch, RedPath 1998 : E. HATCh, H.A. RedPath, A Concordance to the Septuagint and the Other Versions of the Old Testament (Including the Apocryphal Books), $2^{\mathrm{e}}$ éd., Grand Rapids.

KIENAST 2001 : B. KIENAST, Historische semitische Sprachwissenschaft, Wiesbaden.

KINDT 2004 : B. KINDT, « La lemmatisation des sources patristiques et byzantines au service d'une description lexicale du grec ancien. Les principes de formulation des lemmes du Dictionnaire Automatique Grec », Byzantion, 74, p. 213-272 [pdf].

KINDT 2018 : B. KINDT, « Processing Tools for Greek and Other Languages of the Christian Middle East », dans jdmdh:4184 - Journal of Data Mining \& Digital Humanities, January 6, 2018. Special Issue on Computer-Aided Processing of Intertextuality in Ancient Languages [pdf].

KIRAZ 1993 : G.A. KIRAZ, A Computer-Generated Concordance to the Syriac New Testament, 6 vol., Leiden, New York, Cologne.

KIRAZ 2001 : G.A. KIRAZ, MELTHO : Syriac OpenType Fonts for Windows XP/2000 ${ }^{\mathrm{TM}}$ and Windows 95/98/ME ${ }^{\mathrm{TM}}$, Piscataway, 2000-2001 [pdf].

LEFER à paraître : M.-A. LEFER, « Parallel Corpora », à paraître dans S. GRIES, M. Paquot (éd.), Practical Handbook of Corpus Linguistics, New York.

LIPIŃSKI 2000 : E. LIPIŃSKI, Semitic Languages: Outline of a Comparative Grammar (Orientalia Lovaniensia Analecta, 80), Leuven.

LUND 2007 : A.J. LunD, The Book of the laws of the countries: a dialogue on free will versus fate : a key-word-in-context concordance, Piscataway.

Lund, Kiraz 2004 : J.A. Lund, G.A. KiraZ, The Old Syriac Gospel of the Distinct Evangelists: a Key-word-in-context Concordance, Piscataway.

Moscati 1980 : S. Moscati, An Introduction to the Comparative Grammar of the Semitic Languages. Phonology and Morphology (Porta Linguarum Orientalium, NS 6), Wiesbaden.

Mossay et alii 1990 : J. Mossay, Cetedoc, Thesaurus sancti Gregorii Nazianzeni, vol. I. Enumeratio lemmatum, orationes, epistulae, testamentum (Corpus Christianorum. Thesaurus Patrum Graecorum), Turnhout.

Mossay et alii 1991 : J. Mossay, B. Coulie, Cetedoc, Thesaurus sancti Gregorii Nazianzeni, vol. II. Enumeratio lemmatum, carmina, christus patiens, vita (Corpus Christianorum. Thesaurus Patrum Graecorum), Turnhout.

NÖLDEKE 1898 : Th. NöLDEKE, Kurzgefasste syrische Grammatik, Leipzig (réimpr. Darmstadt, 2015).

PATARIDZE, Kindt 2018 : T. PATARIDZe, B. KindT, « Text Alignment in Ancient Greek and Georgian: A Case-Study on the First Homily of Gregory of Nazianzus », dans jdmdh:4182 - Journal of Data Mining \& Digital Humanities, January 6, 2018. Special Issue on Computer-Aided Processing of Intertextuality in Ancient Languages [pdf].

Payne Smith 1967 : J. Payne Smith, A Compendious Syriac Dictionary, founded upon the Thesaurus Syriacus of R. Payne Smith, Oxford, $3^{\mathrm{e}}$ ed. 
Payne Smith 1901 : R. Payne Smith, Thesaurus Syriacus, Oxford, 1879-1901 (réimpr. Hildesheim, New York, 2006).

SCHMIDT 2002 : A.B. SCHMIDT, Sancti Gregorii Nazianzeni Opera. Versio Syriaca. II. Orationes XIII, XLI (Corpus Christianorum. Series Graeca, 47 ; Corpus Nazianzenum, 15), Turnhout.

Sembiante 2017 : A.S. Sembiante, « Appunti sulla tradizione siriaca delle opere di Gregorio Nazianzeno », Koininia 10, p. 607-635.

Sokoloff 2009 : M. SoKoloff, A Syriac Lexicon. A Translation from the Latin, Correction, Expansion, and Update of C. Brockelmann's Lexicon Syriacum, Winona Lake, Piscataway.

TUERLINCKX 2004 : L. TUERLINCKX, « La lemmatisation de l'arabe non classique », dans A. Dister, C. FAiron, G. PURNelle (éd.), Le poids des mots. $7^{e s}$ Journées internationales d'Analyse statistique des Données Textuelles, 10-12 mars 2004, Louvain-la-Neuve, Louvain-la-Neuve, vol. II, p. 1069-1078 [pdf].

VAn Elverdinghe 2018 : E. Van Elverdinghe, « Recurrent Pattern Modelling in a Corpus of Armenian Manuscript Colophons », dans jdmdh:4183 - Journal of Data Mining \& Digital Humanities, January 6, 2018, Special Issue on Computer-Aided Processing of Intertextuality in Ancient Languages [pdf].

Winter 1976 : M.M. Winter, A Concordance to the Peshitta Version of Ben Sira (Monographs of the Peshitta Institute Leiden, 2), Leiden. 